\title{
On a uniform approximation of motion by anisotropic curvature by the Allen-Cahn equations
}

\author{
YoshikAZU GIGA ${ }^{\dagger}$ AND TAKESHI OHTSUKA ${ }^{\ddagger}$ \\ Graduate School of Mathematical Sciences, University of Tokyo, \\ Komaba 3-8-1, Tokyo 153-8914, Japan \\ REINER SCH ̈̈TZLE ${ }^{\S}$ \\ Mathematisches Institut der Eberhard-Karls-Universität Tübingen, \\ Auf der Morgenstelle 10, D-72076 Tübingen, Germany
}

[Received 7 July 2005 and in revised form 21 April 2006]

\begin{abstract}
The convergence of solutions of anisotropic Allen-Cahn equations is studied when the interface thickness parameter (denoted by $\varepsilon$ ) tends to zero. It is shown that the convergence to a level set solution of the corresponding anisotropic interface equations is uniform with respect to the derivatives of a surface energy density function. As an application the crystalline motion of interfaces is shown to be approximated by the anisotropic Allen-Cahn equations.
\end{abstract}

2000 Mathematics Subject Classification: 35B25, 35K57, 53C44.

Keywords: Anisotropic Allen-Cahn equation; anisotropic mean curvature flow; viscosity solution; crystalline curvature flow.

\section{Introduction}

In this paper we consider the anisotropic Allen-Cahn equation with kinetic term. The convergence of solutions of anisotropic Allen-Cahn equations is already proved in [ElS1] (in case the kinetic term is isotropic), [EIPS], and [EIS2]. However, their estimate of the convergence depends on the derivative of the surface energy density function which expresses the anisotropy of the equilibrium form of interfaces. In this paper we obtain a uniform estimate of the convergence with respect to the derivatives of the surface energy density function. One of applications of our result is approximation of the crystalline motion of interfaces by anisotropic Allen-Cahn equations.

The anisotropic Allen-Cahn equation is proposed in [MWBCS]. We consider a functional of the form

$$
F_{\varepsilon}(v)=\int_{\mathbb{R}^{n}}\left[\frac{1}{2} \gamma(\nabla v)^{2}+\frac{1}{\varepsilon^{2}}(W(v)-\varepsilon \lambda f v)\right] \mathrm{d} x .
$$

Here $\gamma \in C^{2}\left(\mathbb{R}^{n} \backslash\{0\}\right)$ is positive on $S^{n-1}$, convex, positively homogeneous of degree one. Moreover, we assume that $\gamma^{2}$ is strictly convex. The function $W$ is a double-well potential of the form $W(v)=\left(v^{2}-1\right)^{2} / 2$. The quantity $\lambda$ is a normalized constant determined by $W$. The quantity

\footnotetext{
${ }^{\dagger}$ E-mail: labgiga@ms.u-tokyo.ac.jp

E-mail: tohtsuka@ms.u-tokyo.ac.jp

${ }^{\S}$ E-mail: schaetz@everest.mathematik.uni-tuebingen.de
} 
$f$ is a given constant. We consider the weighted $L^{2}$-gradient flow of this functional, and obtain the anisotropic Allen-Cahn equation. Its explicit form is

$$
\beta(\nabla v) \partial_{t} v-\operatorname{div} \gamma(\nabla v) \xi(\nabla v)+\frac{1}{\varepsilon^{2}}\left(W^{\prime}(v)-\varepsilon \lambda f\right)=0
$$

Here $\beta \in C\left(\mathbb{R}^{n} \backslash\{0\}\right)$ is positive on $S^{n-1}$ and positively homogeneous of degree zero, and $\xi=$ $D \gamma=\left(\partial_{p_{1}} \gamma(p), \ldots, \partial_{p_{n}} \gamma(p)\right)$ for $p=\left(p_{1}, \ldots, p_{n}\right)$. A formal asymptotic analysis provided by [MWBCS], [WM] and [BP1] (the case $\beta \equiv 1$ ) says that the internal transition layer of (1.1) approximates the evolving interface $\left\{\Gamma_{t}\right\}_{t} \geqslant 0$ under the evolution law of the form

$$
\beta(\mathbf{n}) V=-\gamma(\mathbf{n})\left\{\operatorname{div}_{\Gamma_{t}} \xi(\mathbf{n})+f\right\} \quad \text { on } \Gamma_{t},
$$

where $\mathbf{n}$ denotes the outer unit normal vector field of $\Gamma_{t}, V$ denotes the normal velocity in the direction of $\mathbf{n}$, and $\operatorname{div}_{\Gamma_{t}}$ is the surface divergence on $\Gamma_{t}$. The constant $\lambda$ is taken so that the multiplicative constant in front of $f$ in 1.2 equals one. Physically, the function $\gamma$ is called the surface energy density, which expresses the anisotropy of the equilibrium form of interfaces. The function $\xi$ is called the Cahn-Hoffman vector. The function $\beta$ expresses the anisotropy of kinetics. The quantity $f$ is a driving force of the evolution. The quantity $\gamma / \beta$ is called mobility.

If the initial data $v(x, 0)$ of $(1.1)$ is positive in a region $O_{0}$ enclosed by $\Gamma_{0}$ and negative in $\mathbb{R}^{n} \backslash\left(O_{0} \cup \Gamma_{0}\right)$, then one expects that

$$
v \rightarrow \begin{cases}+1 & \text { in a region } O_{t} \text { enclosed by } \Gamma_{t}, \\ -1 & \text { in } \mathbb{R}^{n} \backslash\left(O_{t} \cap \Gamma_{t}\right),\end{cases}
$$

locally uniformly as $\varepsilon \rightarrow 0$. This fact is rigorously proved in [ElS1] locally in time at least if the initial interface is smooth. Using a level set method due to [CGG1] and [ES] the authors of [ElPS] and [ElS2] proved (1.3) globally-in-time by interpreting $\Gamma_{t}$ as a generalized solution of (1.2). They introduced a signed anisotropic distance function from $\Gamma_{t}$ as outlined in [BP2] (see Section 3). By using this distance, they constructed a sub- and supersolution of (1.1) to prove the convergence (1.3.

We note that the convergence results in [EIS2] depend on the smoothness of $\gamma$ : the way to determine $\varepsilon$ for the estimate to obtain (1.3) depends at least on the second derivatives of $\gamma$. Physically, however, it may happen that $\gamma$ is not smooth so that the equilibrium form of the interface of (1.2) may have a flat portion called a facet. If one tries to consider such a situation by (1.1) with $\gamma_{a}$ approximating nonsmooth $\gamma$, their results are not enough.

In this paper we will show that the convergence of the internal transition layers is in some sense 'uniform' with respect to the derivatives of $\gamma$ provided that $\gamma, 1 / \gamma, \beta$, and $1 / \beta$ on the unit sphere are bounded. No control of the derivatives of $\gamma$ is necessary. This gives a way to approximate the crystalline motion $[\mathrm{T}],[\mathrm{AG}]$ in the plane by an anisotropic Allen-Cahn type equation in conjunction with a general level set method for nondifferentiable $\gamma$ in [GG4], [GG5]. This will be explained in $\$ 2.5$ as an application of our main result. In [BGN] the anisotropic Allen-Cahn equation with crystalline $\gamma$ and $\beta \equiv 1$ in $\mathbb{R}^{2}$ is considered, and it is extended to the problem in $\mathbb{R}^{n}$ for $n \geqslant 2$ in $[\overline{\mathrm{BN}}]$. They derived even a convergence rate of the internal layer of the Allen-Cahn equation when the limit evolution is a crystalline motion. By the assumption $\beta \equiv 1,2.6$ is considered as a variational inequality. (Several examples of solutions are proposed in [TC].) Although we mollify $\gamma$ and $\beta$, one advantage of our theory is that anisotropic $\beta$ can be handled. We approximate nonsmooth 
$\gamma$ by smoother $\gamma^{\tau}$ while [BGN] studied the Allen-Cahn equation with nonsmooth $\gamma$ by using a subdifferential.

The difficulty in treating (1.1) directly is that (1.1) does not enjoy a comparison principle. This is caused by singularities at $\nabla v=0$ which are due to the nonconstant kinetic factor $\beta$. This difficulty is overcome in [EIS2] by adjusting a definition of solution to have a comparison principle. In this paper we overcome the difficulty caused by singularities of $\beta$ in another way. We introduce a modified equation in place of (1.1) to remove singularities. The advantage of our idea is that the usual theory of viscosity solutions is available for the modified equation. We prove that the solution of the modified equation satisfies (1.3) and the convergence is 'uniform' with respect to the derivatives of $\gamma$.

The basic strategy of the proof of [1.3) is a combination of the method of [ESS] and [ElS2]. However, we need to estimate the time derivative of the anisotropic distance function in a different way. We construct a viscosity supersolution of (1.1) for an estimate to obtain the convergence result by combining three ingredients: a distance function induced by Finsler geometry as in [BP2], its truncation as in [ESS] and the traveling wave as in [BSS]. The key reason why we are able to prove the uniform convergence result with respect to the modulus of the derivative of $\gamma$ is an estimate of the time derivative of the distance function from $\Gamma_{t}$. Although the time derivative is estimated in [EIS2], their bound depends on the second derivatives of $\gamma$ on $S^{n-1}$. In this paper we will prove such an estimate by using a duality between $\gamma$ and the support function of $\left\{p \in \mathbb{R}^{n} ; \gamma(p) \leqslant 1\right\}$ so that no derivatives of $\gamma$ are involved.

Recently, $[\overline{B S}]$ and $[\overline{B D L}]$ provided the geometrical approach to approximating the motion of interfaces. However, their method does not provide our uniform convergence.

Finally, we note that, for the isotropic case $(\beta(p) \equiv 1, \gamma(p)=|p|)$, the convergence problem has been extensively studied in various contexts, e.g., [BK], [DS], [C], [ESS], [BSS], [I], [So], etc.

\section{Main result}

\subsection{Equations}

We now recall the anisotropic mean curvature flow. Let $\left\{\Gamma_{t}\right\}_{t} \geqslant 0$ be a family of closed hypersurfaces in $\mathbb{R}^{n}$. We consider the evolution law for $\Gamma_{t}$ of the form

$$
\beta(\mathbf{n}) V=-\gamma(\mathbf{n})\left\{\operatorname{div}_{\Gamma_{t}} \xi(\mathbf{n})+f\right\} \quad \text { on } \Gamma_{t},
$$

where $V$ denotes the normal velocity of the surface $\Gamma_{t}$ and $\mathbf{n}$ denotes the outer unit normal vector field of $\Gamma_{t}$. In this paper we assume that

( $\beta 1) \quad \beta \in C\left(\mathbb{R}^{n} \backslash\{0\}\right)$,

( $\beta 2) \beta$ is positively homogeneous of degree 0 ,

( $\beta 3$ ) there exists a positive constant $\Lambda_{\beta}$ satisfying $\Lambda_{\beta}^{-1} \leqslant \beta \leqslant \Lambda_{\beta}$ on $S^{n-1}$,

$(\gamma 1) \gamma \in C^{2}\left(\mathbb{R}^{n} \backslash\{0\}\right)$

$(\gamma 2) \gamma$ is positively homogeneous of degree 1 ,

( $\gamma 3)$ there exists a positive constant $\Lambda_{\gamma}$ satisfying $\Lambda_{\gamma}^{-1} \leqslant \gamma \leqslant \Lambda_{\gamma}$ on $S^{n-1}$,

$(\gamma 4) \gamma$ is convex,

$(\gamma 5) \alpha:=\gamma^{2} / 2$ is strictly convex,

( $f 1) f$ is a given constant satisfying $|f| \leqslant \Lambda_{f}$ with some $\Lambda_{f}>0$,

( $\varepsilon 1) \varepsilon \in(0, \bar{\varepsilon})$, where $\bar{\varepsilon}$ is such that the function $\sigma \mapsto W^{\prime}(\sigma)-\varepsilon \lambda \Lambda_{f}$ has exactly three zeros, 
where $S^{n-1}$ is a unit sphere. The vector field $\xi$ is the gradient field of $\gamma$, i.e., $\xi=D \gamma=$ $\left(\partial_{p_{1}} \gamma, \ldots, \partial_{p_{n}} \gamma\right), \partial_{p_{i}} \gamma=\partial \gamma / \partial p_{i}, 1 \leqslant i \leqslant n$. The divergence operator in 2.1) is the surface divergence on $\Gamma_{t}$. In this paper, we only consider the driving force term $f$ that is constant.

A level set formulation for 2.1) gives a generalized notion of the motion of $\Gamma_{t}$ (see [CGG1], [CGG2], [G1]). We introduce an auxiliary function $u: \mathbb{R}^{n} \times[0, T) \rightarrow \mathbb{R}$ and define

$$
\Gamma_{t}=\left\{x \in \mathbb{R}^{n} ; u(x, t)=0\right\} .
$$

The level set equation obtained from $(2.1)$ is of the form

$$
\beta(\nabla u) \partial_{t} u-\gamma(\nabla u)\{\operatorname{div} \xi(\nabla u)+f\}=0 \quad \text { in } \mathbb{R}^{n} \times(0, T) .
$$

Here div denotes the divergence in $\mathbb{R}^{n}$, and $\nabla$ denotes the spatial derivatives, i.e., $\nabla v=$ $\left(\partial_{x_{1}} v, \ldots, \partial_{x_{n}} v\right)$, so we distinguish between the differential operator $D$ and the spatial derivative $\nabla$. We say that $\left\{\Gamma_{t}\right\}_{t \in[0, T)}$ is a generalized solution of (2.1) if $\Gamma_{t}$ is given by (2.2) for an auxiliary function $u \in C\left(\mathbb{R}^{n} \times[0, T)\right)$ which is a viscosity solution of $(2.3)$.

We are interested in the motion of $\Gamma_{t}$, which starts from some compact $\Gamma_{0}$, in a finite time interval $(0, T)$. Then, since any viscosity solution of $(2.3)$ with continuous initial data is continuous, we may assume that there exists a big cube $\prod_{j=1}^{n}\left[a_{j}, b_{j}\right]$ satisfying $\Gamma_{t} \subset \prod_{j=1}^{n}\left[a_{j}, b_{j}\right]$ for $t \in$ $[0, T)$. Therefore we consider the equation with the periodic boundary condition, i.e., the equality $u\left(x+\left(b_{j}-a_{j}\right) e_{j}, t\right)=u(x, t)$ holds for $(x, t) \in \mathbb{R}^{n} \times[0, T)$ and $j=1, \ldots, n$. We now set $\mathbb{T}^{n}=\prod_{j=1}^{n} \mathbb{R} /\left(b_{j}-a_{j}\right) \mathbb{Z}$. We consider 2.3$)$ on $\mathbb{T}^{n} \times(0, T)$, i.e.,

$$
\beta(\nabla u) \partial_{t} u-\gamma(\nabla u)\{\operatorname{div} \xi(\nabla u)+f\}=0 \quad \text { in } \mathbb{T}^{n} \times(0, T)
$$

with initial data

$$
u(\cdot, 0)=u_{0}(\cdot) \quad \text { on } \mathbb{T}^{n} .
$$

Since (2.4) is degenerate parabolic and geometric, it is well known that, for periodic initial data, there exists a unique global periodic viscosity solution of [2.4] (see [CGG1], [GGIS] or [G2]).

There is another way to analyze the motion of $\Gamma_{t}$. In fact, there is the approximation of $\Gamma_{t}$ by the internal transition layer of an anisotropic Allen-Cahn type equation introduced in [MWBCS]. The explicit form of the equation is

$$
\beta(\nabla v) \partial_{t} v-\operatorname{div}\{\gamma(\nabla v) \xi(\nabla v)\}+\frac{1}{\varepsilon^{2}}\left(W^{\prime}(v)-\varepsilon \lambda f\right)=0 \quad \text { in } \mathbb{T}^{n} \times(0, T),
$$

with initial data

$$
v(\cdot, 0)=v_{0}(\cdot) \quad \text { on } \mathbb{T}^{n} .
$$

Here $W$ is a double-well potential of the form $W(\sigma)=\left(\sigma^{2}-1\right)^{2} / 2$, and $\lambda$ is a constant determined by $W$, in our case $\lambda=2 / 3$. We choose a suitable $v_{0}$ to approximate an interface moving by 2.1). See Section 2.4 and Theorem 2.2 for the choice of $v_{0}$. The internal transition layers of (2.6) approximate the motion of $\Gamma_{t}$. This fact is already established rigorously in [ElS1], [ElPS] and [ElS2].

Our aim in this paper is to prove that an estimate of the convergence of internal transition layers is uniform with respect to the modulus of derivatives of $\gamma$. For this purpose, we have to specify the quantities which determine the speed of the convergence of internal transition layers.

Traditionally as in [EIS2] or [ESS], we construct a supersolution and a subsolution of (2.6) to estimate the convergence. The key tool of this method is the comparison principle for viscosity 
solutions. Unfortunately, however, 2.6 has singularities so that we cannot apply the usual comparison principle for viscosity solutions. To overcome this difficulty, we modify the equation. We introduce a cut-off function $\zeta \in C^{\infty}([0, \infty))$ satisfying

$$
\zeta(\sigma)= \begin{cases}1 & \text { if } \sigma \leqslant 1 / 2 \\ 0 & \text { if } \sigma \geqslant 3 / 4\end{cases}
$$

and $\zeta^{\prime} \leqslant 0$. Let $\tilde{\beta}$ be a function defined by

$$
\tilde{\beta}(p)=(1-\zeta(|p|)) \beta(p)+\Lambda_{\beta} \zeta(|p|) .
$$

We replace the coefficient $\beta(\nabla v)$ of $\partial_{t} v$ in 2.6 by $\tilde{\beta}(\nabla v)$, i.e.,

$$
\tilde{\beta}(\nabla v) \partial_{t} v-\operatorname{div}\{\gamma(\nabla v) \xi(\nabla v)\}+\frac{1}{\varepsilon^{2}}\left(W^{\prime}(v)-\varepsilon \lambda f\right)=0 \quad \text { in } \mathbb{T}^{n} \times(0, T) .
$$

The same type of modification appears in [EIPS]. The main advantage of 2.9] over 2.6) is that the singularity at $\nabla v=0$ in the term involving $\beta$ is removed. Since $\widetilde{\beta}$ is positive and continuous on $\mathbb{R}^{n}$, we can apply the usual theory of viscosity solutions, in particular the comparison principle (see [CGG1] or [G2]). We treat (2.9] as an approximate model of an anisotropic mean curvature flow instead of (2.6). The solvability of 2.9) with initial data $v_{0} \in C\left(\mathbb{T}^{n}\right)$ is already established in [EIS2, Section 2.3 and Theorem 2.8] (see also [LSU|).

\subsection{Anisotropic distance function}

We now recall the anisotropic distance function induced by a Finsler (Minkowski) metric as in [BP2]. The distance is useful to construct an initial datum for 2.4p or 2.9).

We define the support function $\gamma^{\circ}$ of the convex set $\left\{p \in \mathbb{R}^{n} ; \gamma(p) \leqslant 1\right\}$ by

$$
\gamma^{\circ}(p)=\sup \{\langle p, q\rangle ; \gamma(q) \leqslant 1\}
$$

Note that $\gamma^{\circ} \in C^{2}\left(\mathbb{R}^{n} \backslash\{0\}\right)$, and $\gamma^{\circ}$ is convex, positively homogeneous of degree 1 . Moreover, for $p \in \mathbb{R}^{n} \backslash\{0\}$, there exists a unique $q \in\left\{p \in \mathbb{R}^{n} ; \gamma(p) \leqslant 1\right\}$ satisfying $\gamma^{\circ}(p)=\langle p, q\rangle$ since $\gamma^{2}$ is strictly convex. Properties of $\gamma^{\circ}$ important for studying [2.6) or [2.9] are obtained in [BP2]. We shall list some of them in Section 3.

We define an anisotropic distance $\Xi$ by

$$
\Xi(x, y)=\gamma^{\circ}(x-y) .
$$

We remark that only the symmetry in the definition of distance does not hold for $\Xi$ since $\gamma^{\circ}$ is not assumed to be symmetric. For a subset $\Gamma \subset \mathbb{R}^{n}$ we define

$$
\Xi(x, \Gamma)=\inf \{\Xi(x, y) ; y \in \Gamma\} .
$$

The following argument also applies to the reversed version of the anisotropic distance function of the form $\Xi(\Gamma, x)$. 


\subsection{Traveling wave}

To derive an estimate for the convergence of the internal transition layer of (2.9), it is convenient to introduce a traveling wave solution of 2.9 with initial data which has a layer around $\Gamma_{0}$. In general, we consider a solution of 2.9) of the form $v(x, t)=Q(x \cdot e-c t)$ for the function $Q$, constant $c$ and fixed $e \in S^{n-1}$. Then we observe that $Q$ satisfies some ordinary differential equation. However, here it suffices to consider the equation of $Q$ for the isometric case as in [BSS].

Now we introduce a generalized notion of a traveling wave. We shall consider the double-well potential of the form $W(\sigma)-z \sigma$ for $z \in \mathbb{R}$. For $z$ with $|z|<4 \sqrt{3} / 9$, the function $\sigma \mapsto W^{\prime}(\sigma)-z$ has exactly three zeros. We shall denote them by $h_{-}=h_{-}(z), h_{0}=h_{0}(z)$ and $h_{+}=h_{+}(z)$, where $h_{-}<h_{0}<h_{+}$. We assume that $z$ satisfies $|z|<4 \sqrt{3} / 9$, and set

$$
\begin{aligned}
m(z) & =h_{+}(z)-h_{-}(z), \\
c(z) & =2 h_{0}(z)-\left(h_{+}(z)+h_{-}(z)\right), \\
Q(\sigma, z) & =h_{-}(z)+\frac{m(z)}{1+\exp \left\{-m(z)\left(\sigma-\sigma_{0}(z)\right)\right\}},
\end{aligned}
$$

where $\sigma_{0}(z)$ is taken so that $Q$ satisfies $Q(0, z)=h_{0}(z)$. Since $h_{ \pm}$and $h_{0}$ are smooth, we observe that $Q \in C^{\infty}(\mathbb{R} \times(-4 \sqrt{3} / 9,4 \sqrt{3} / 9))$ and it solves

$$
\begin{aligned}
& Q_{\sigma \sigma}(\sigma, z)+c(z) Q_{\sigma}(\sigma, z)=W^{\prime}(Q(\sigma, z))-z \quad \text { for } \sigma \in \mathbb{R}, \\
& \lim _{\sigma \rightarrow \pm \infty} Q(\sigma, z)=h_{ \pm}(z), \quad Q(0, z)=h_{0}(z) .
\end{aligned}
$$

Moreover,

$$
\begin{gathered}
h_{ \pm}(z)= \pm 1+O(z), \quad h_{0}(z)=0+O(z) \\
m(z)=2+O\left(z^{2}\right), \quad \text { in particular } \quad \sqrt{3}<m(z) \leqslant 2 \\
\frac{c(z)}{z}=\frac{2}{W^{\prime \prime}(0)}-\left(\frac{1}{W^{\prime \prime}(1)}+\frac{1}{W^{\prime \prime}(-1)}\right)+O(z)=-\frac{1}{\lambda}+O(z)
\end{gathered}
$$

as $z \rightarrow 0$.

In our case we fix $z=\varepsilon \lambda f$ and set $Q(\sigma)=Q(\sigma, \varepsilon \lambda f)$. Hereafter we omit the dependence on $z$ when $z=\varepsilon \lambda f$, and we write $Q^{\prime}(\sigma)=Q_{\sigma}(\sigma, \varepsilon \lambda f)$ and $Q^{\prime \prime}(\sigma)=Q_{\sigma \sigma}(\sigma, \varepsilon \lambda f)$. We list some properties of these functions.

Proposition 2.1 Assume that $f$ satisfies $(f 1)$ and $\varepsilon$ satisfies $(\varepsilon 1)$. Then:

(i) $\lim _{\varepsilon \rightarrow 0} \sup _{|f| \leqslant \Lambda_{f}}|c / \varepsilon+f|=0$,

(ii) $\lim _{\varepsilon \rightarrow 0} \sup \left\{|Q(\sigma)-\tanh \sigma| ; \sigma \in \mathbb{R}, f \in\left[-\Lambda_{f}, \Lambda_{f}\right]\right\}=0$,

(iii) $\inf \left\{Q^{\prime}(\sigma) ; \sigma \in[-b, b], \varepsilon \in(0, \bar{\varepsilon}), f \in\left[-\Lambda_{f}, \Lambda_{f}\right]\right\}>0$ for $b>0$,

(iv) there exist constants $C_{1}, C_{2}$ and $C_{3}$, which depend only on $\Lambda_{f}$, satisfying

$$
\begin{gathered}
\left|Q(\sigma)^{2}-1\right| \leqslant C_{1} \exp \left(-C_{2}|\sigma|\right)+C_{3} \varepsilon \\
\left|Q^{\prime}(\sigma)\right|,\left|Q^{\prime \prime}(\sigma)\right| \leqslant C_{1} \exp \left(-C_{2}|\sigma|\right) .
\end{gathered}
$$




\subsection{Main result}

We now determine the interfaces moving by 2.1. Let $O_{0}$ be an open subset in $\mathbb{T}^{n}$ and $\Gamma_{0}=\partial O_{0}$. Let $d_{0}$ be a signed anisotropic distance function from an initial interface $\Gamma_{0}$ defined by

$$
d_{0}(x)= \begin{cases}\Xi\left(x, \Gamma_{0}\right) & \text { if } x \in O_{0} \cup \Gamma_{0}, \\ -\Xi\left(x, \Gamma_{0}\right) & \text { otherwise }\end{cases}
$$

We note that $d_{0}$ is continuous on $\mathbb{T}^{n}$ and spatially periodic. Let $u$ be a periodic viscosity solution of 2.4 with initial data $u_{0}=d_{0}$. Then we obtain a generalized solution $\Gamma_{t}$ of (2.1) starting from $\Gamma_{0}$, defined by 2.2.

We assume that $\Gamma_{t} \neq \emptyset$ for $t \in[0, T)$. We define a signed anisotropic distance function $d: \mathbb{T}^{n} \times$ $[0, T) \rightarrow \mathbb{R}$ from $\Gamma_{t}$ by

$$
d(x, t)= \begin{cases}\Xi\left(x, \Gamma_{t}\right) & \text { if } x \in\left\{y \in \mathbb{T}^{n} ; u(y, t) \geqslant 0\right\} \\ -\Xi\left(x, \Gamma_{t}\right) & \text { if } x \in\left\{y \in \mathbb{T}^{n} ; u(y, t)<0\right\}\end{cases}
$$

We are now in a position to state our main result.

Theorem 2.2 Assume that $\beta, \gamma, f$, and $\varepsilon$ satisfy $(\beta 1)-(\beta 3),(\gamma 1)-(\gamma 5),(f 1)$, and $(\varepsilon 1)$ respectively. Let $O_{0}$ be an open set in $\mathbb{T}^{n}$ and $\Gamma_{0}=\partial O_{0}$. Let $d_{0}, d(x, t)$ be the anisotropic signed distance functions from $\Gamma_{0}, \Gamma_{t}$ defined by 2.13, 2.14), respectively. Let $v$ be a viscosity solution of (2.9) satisfying (2.8) with initial data $v_{0}(x)=Q\left(d_{0}(x) / \varepsilon\right)$ for $\varepsilon<\bar{\varepsilon}$. For $\theta>0$, there exist positive constants $\delta=\delta(\theta), \varepsilon_{1}=\varepsilon_{1}\left(\theta, \Lambda_{\beta}, \Lambda_{\gamma}, \Lambda_{f}\right)$ and $C=C\left(\theta, \Lambda_{\beta}, \Lambda_{\gamma}, \Lambda_{f}\right)$ satisfying

$$
v(x, t) \leqslant-1+C_{1} \exp \left(-\frac{C_{2} \delta}{\varepsilon}\right)+C \varepsilon
$$

if $(x, t) \in\left\{(y, s) \in \mathbb{T}^{n} \times(0, T) ; d(y, s) \leqslant-\theta\right\}$ provided that $\varepsilon \in\left(0, \varepsilon_{1}\right)$, where $C_{1}$ and $C_{2}$ are numerical constants.

This result is a refined version of [EIS2] since the constants $C_{1}, C_{2}, C$ and $\varepsilon_{1}$ are independent of the first and second derivatives of $\gamma$. This is useful to treat the approximating problem of (2.4) and (2.9) for nonsmooth $\gamma$.

The main strategy of the proof comes from [ESS] and [ElS2]. We construct a function $\psi=\psi_{\varepsilon, \delta}$ satisfying:

(i) for $\theta>0$, there exist positive constants $\delta=\delta(\theta)$ and $C=C\left(\theta, \Lambda_{\beta}, \Lambda_{\gamma}\right)$ such that $\psi(x, t)$ satisfies 2.15) for $(x, t) \in\left\{(y, s) \in \mathbb{T}^{n} \times(0, T) ; d(y, s)<-\theta\right\}$,

(ii) for this $\delta$, there exists a positive constant $\varepsilon_{0}$ such that $\psi$ is a supersolution of (2.9) provided that $\varepsilon \in\left(0, \varepsilon_{0}\right)$,

(iii) $\psi(x, 0) \geqslant Q\left(d_{0}(x) / \varepsilon\right)$.

Then, by the comparison principle, we obtain Theorem 2.2. Unfortunately the construction of [ESS] and [ElS2] is suitable only to construct a supersolution of the unmodified equation [2.6, but not of 2.9. To clarify the difficulty in obtaining (i) we shall give a formal calculation. Set

$$
\begin{aligned}
& R_{\varepsilon}=\beta(\nabla \psi)-\operatorname{div}\{\gamma(\nabla \psi) \xi(\nabla \psi)\}+\frac{1}{\varepsilon^{2}}\left(W^{\prime}(\psi)-\varepsilon \lambda f\right), \\
& \tilde{R}_{\varepsilon}=\tilde{\beta}(\nabla \psi)-\operatorname{div}\{\gamma(\nabla \psi) \xi(\nabla \psi)\}+\frac{1}{\varepsilon^{2}}\left(W^{\prime}(\psi)-\varepsilon \lambda f\right) .
\end{aligned}
$$


Clearly the first quantity $R_{\varepsilon}$ is easy to calculate. However, we have to calculate $\tilde{R}_{\varepsilon}$. We observe that

$$
\tilde{R}_{\varepsilon}=R_{\varepsilon}+\left(\Lambda_{\beta}-\beta(\nabla \psi)\right) \zeta(|\nabla \psi|) \partial_{t} \psi .
$$

Thus it suffices to derive a suitable estimate for $\partial_{t} \psi$ to calculate $\tilde{R}_{\varepsilon}$.

We summarize the way of constructing $\psi$ :

(i) (\$3.1 and §3.2) We verify that the anisotropic signed distance function $d$ is a viscosity supersolution of 2.4 in $\left\{(x, t) \in \mathbb{R}^{n} \times(0, T) ; d(x, t)>0\right\}$. We also give an estimate of $\partial_{t} d$.

(ii) (§3.3) For fixed $\delta$, we introduce a truncating function $\eta$ as in $[\mathrm{ESS}]$ and consider $\omega=\eta(d)$. We give an estimate of $\beta(\nabla \omega) \partial_{t} \omega-\operatorname{div}\{\gamma(\nabla \omega) \xi(\nabla \omega)\}$. We also give an estimate of $\partial_{t} \omega$.

(iii) $(\S 4)$ We construct a function $\psi$ by using $\omega$. We verify that, for $\delta$, there exists a positive constant $\varepsilon_{0}=\varepsilon_{0}\left(\delta, \Lambda_{\beta}, \Lambda_{\gamma}\right)$ such that $\psi$ is a viscosity supersolution of 2.6 provided that $\varepsilon \in\left(0, \varepsilon_{0}\right)$.

(iv) (§4) We verify that, for $\delta$, there exists a positive constant $\varepsilon_{1}=\varepsilon_{1}\left(\delta, \Lambda_{\beta}, \Lambda_{\gamma}\right)$ such that $\psi$ is a viscosity supersolution of $(2.9)$ provided that $\varepsilon \in\left(0, \varepsilon_{1}\right)$.

We give the proof of Theorem 2.2 in $\$ 5$.

Hereafter, we often use another representation of the second terms of (2.4), (2.6) and (2.9), i.e.,

$$
\operatorname{div}\{\xi(\nabla u)\}=\operatorname{tr}\left\{D^{2} \gamma(\nabla u) \nabla^{2} u\right\}, \quad \operatorname{div}\{\gamma(\nabla v) \xi(\nabla v)\}=\operatorname{tr}\left\{D^{2} \alpha(\nabla v) \nabla^{2} v\right\},
$$

where $\alpha(p)=\gamma(p)^{2} / 2$. We remark that $\alpha$ is positively homogeneous of degree 2 .

Finally, we remark that we only estimate solutions from above. This is because estimation from below is essentially the same by considering (2.6) and (2.9) with $\tilde{\beta}(p)=\beta(-p), \tilde{\alpha}(p)=\alpha(-p)$, $\tilde{W}(\sigma)=W(-\sigma)$, and $\tilde{f}=-f$ instead of $\beta(p), \alpha(p), W(\sigma)$, and $f$, respectively. By a standard argument, Theorem 2.2 and this remark yield (1.3).

\subsection{Application}

We now give an application of Theorem 2.2. Our result is useful to approximate solutions of (2.1) by 2.9) even when $\gamma$ is not differentiable provided that 2.4 fulfills the following convergence ansatz.

Convergence Ansatz. Assume that $\beta^{\tau} \in C\left(\mathbb{R}^{n} \backslash\{0\}\right), \gamma^{\tau} \in C^{2}\left(\mathbb{R}^{n} \backslash\{0\}\right)$ are positive and $f^{\tau} \in \mathbb{R}$. Assume that $\beta^{\tau}$ and $\gamma^{\tau}$ are positively homogeneous of degree 0 and 1 , respectively. Assume that $\gamma^{\tau}$ is convex. (We do not assume the differentiability of $\gamma$.) Assume that $\beta^{\tau} \rightarrow \hat{\beta}$, $\gamma^{\tau} \rightarrow \hat{\gamma}$ locally uniformly in $\mathbb{R}^{n} \backslash\{0\}$ and $f^{\tau} \rightarrow \hat{f}$ as $\tau \rightarrow 0$. Let $u^{\tau}$ be the periodic viscosity solution of

$$
\beta^{\tau}\left(\nabla u^{\tau}\right) \partial_{t} u^{\tau}-\gamma^{\tau}\left(\nabla u^{\tau}\right)\left\{\operatorname{div} \xi^{\tau}\left(\nabla u^{\tau}\right)+f^{\tau}\right\}=0 \quad \text { in } \mathbb{R}^{n} \times(0, T),
$$

with continuous periodic initial data $u^{\tau}(x, 0)=u_{0}^{\tau}(x)$, where $\xi^{\tau}=D \gamma^{\tau}$. Assume that the period is independent of $\tau$. Assume that $u_{0}^{\tau} \rightarrow u_{0}$ uniformly in $\mathbb{R}^{n}$. Then $u^{\tau}$ converges to $\hat{u} \in C\left(\mathbb{R}^{n} \times[0, \infty)\right)$ which "solves" 2.4 with $\beta=\hat{\beta}, \gamma=\hat{\gamma}$ and $\hat{u}(x, 0)=u_{0}(x)$. The convergence is uniform in $\mathbb{R}^{n} \times[0, T]$ for every $T>0$.

If we further assume that there exists a function $H \in C\left(S^{n-1} ; \mathbf{S}_{n}\right)$, where $\mathbf{S}_{n}$ denotes the space of real symmetric $n \times n$ matrices, such that $D \xi^{\tau} \rightarrow H$ on $S^{n-1}$, then $\gamma$ is $C^{2}\left(S^{n-1}\right)$ and $D \xi=H$. In this case, the convergence of a solution $u^{\tau}$ to 2.4 with $\beta=\beta^{\tau}, \gamma=\gamma^{\tau}$ and $f=f^{\tau}$ is well 
known (cf. [CGG1], [Ca], and [GG5]). However, if we do not assume the convergence of derivatives of $\gamma^{\tau}$, the convergence ansatz has recently been proved for $n=2$ in [GG4] and [GG5]. Note that the meaning of a solution to (2.4) is not clear at all for nondifferentiable $\gamma$ since the term $\operatorname{div} \xi(\nabla u)$ is not well defined even for smooth $u$. The papers [GG4] and [GG5] provide a proper notion of solution. Note that the notion is consistent with the more restrictive notion of [GG1]; the proof is based on an elementary comparison principle in [GGul.

THEOREM 2.3 ([GG4], [GG5]) Assume that $n=2,\left.\gamma\right|_{S^{1}}$ is $C^{2}$ except at finitely many points $P=$ $\left\{P_{i}\right\}_{i=1}^{m}$, and the angular second derivatives of $\left.\gamma\right|_{S^{1}}$ are bounded on $S^{1} \backslash P$. Then the convergence ansatz is satisfied.

This is a very special version of the results in [GG4] or [GG5], where level set equations for more general equations of the form $V=g\left(\mathbf{n},-\operatorname{div}_{\Gamma_{t}}(\mathbf{n})\right)$ are studied. The main idea of the proof is to reduce the problem to graph-like solutions of [2.1] which are studied in [GG2] and [GG3]. If the convergence ansatz is fulfilled, by a standard argument, Theorem 2.2 yields:

THEOREM 2.4 Assume that the convergence ansatz is true. Assume that $\beta^{\tau}, \gamma^{\tau}$ and $f^{\tau}$ satisfy $(\beta 1)-(\beta 3),(\gamma 1)-(\gamma 5),(f 1)$ and $(\varepsilon 1)$ with $\beta=\beta^{\tau}, \gamma=\gamma^{\tau}$ and $f=f^{\tau}$, uniformly in $\tau$. Let $v^{\tau}$ be a solution of (2.9) with $\beta=\beta^{\tau}, \gamma=\gamma^{\tau}$ and $f=f^{\tau}$ with initial data $v^{\tau}(x, 0)=Q(d(x, 0) / \varepsilon)$. Then

$$
v^{\tau}(x, t) \rightarrow \begin{cases}1 & \text { if } x \in\left\{y \in \mathbb{R}^{n} ; u(y, t)>0\right\}, \\ -1 & \text { if } x \in\left\{y \in \mathbb{R}^{n} ; u(y, t)<0\right\},\end{cases}
$$

as $\tau, \varepsilon \rightarrow 0$. Here $u$ is a solution of 2.4 with $\beta$ and $\gamma$.

Of course, there is always a way to approximate $\gamma$ by $\gamma_{\tau}$ having required properties.

LEMma 2.5 Let $\gamma: \mathbb{R}^{n} \rightarrow \mathbb{R}$ be a function satisfying $(\gamma 2)-(\gamma 4)$. Then there exists a sequence $\left\{\gamma_{\tau}\right\}_{\tau \in\left(0, \tau_{0}\right)}$ of functions satisfying $(\gamma 1)-(\gamma 5)$ such that $\gamma_{\tau} \rightarrow \gamma$ as $\tau \rightarrow 0$ locally uniformly. Moreover, a positive constant $\Lambda_{\gamma}$ in $(\gamma 3)$ for $\gamma_{\tau}$ can be taken independent of $\tau \in\left(0, \tau_{0}\right)$.

REMARK 2.6 The assumption on $\gamma$ in Lemma 2.5 covers cases when $\{p ; \gamma(p)=1\}$ contains nondifferentiable points and/or flat portions. For example, $\gamma(p)=\max \left\{\left|p_{j}\right| ; j=1, \ldots, n\right\}$ or $\gamma(p)=\sum_{j=1}^{n}\left|p_{j}\right|$ satisfies the assumptions of Lemma 2.5

One can find a way to smoothen $\gamma$ in Theorem 3.3.1 of $[\langle\mathrm{Sc}|$. In general, however, approximations to $\gamma$ which are obtained by that method do not satisfy $(\gamma 5)$. In fact, for $\gamma(p)=\max \left\{\left|p_{j}\right| ; j=\right.$ $1, \ldots, n\}$ and small $\tau_{0}$, we have

$$
\gamma_{\tau}(p)=\int_{\mathbb{R}^{n}} \gamma(p+|p| q) \varphi_{\tau}(|q|) \mathrm{d} q=\gamma(p)
$$

in some small neighborhood of $(1,0, \ldots, 0)$, where $\varphi_{\tau}:[0, \infty) \rightarrow[0, \infty)$ is a function as in Theorem 3.3.1 of $[\mathbf{S c}]$ with $\operatorname{supp} \varphi_{\tau} \subset[\tau / 2, \tau]$.

Proof of Lemma 2.5 Here we take the heat kernel $G(p, \tau)=(4 \pi \tau)^{-n / 2} \exp \left(-|p|^{2} / 4 \tau\right)$ and define

$$
\tilde{\gamma}(p, \tau):=(\gamma * G(\cdot, \tau))(p)=\int_{\mathbb{R}^{n}} \gamma(q) G(p-q, \tau) \mathrm{d} q .
$$

We get $\tilde{\gamma} \in C^{\infty}\left(\mathbb{R}^{n} \times(0, \infty)\right)$ and $\tilde{\gamma}(\cdot, \tau) \rightarrow \gamma$ as $\tau \rightarrow 0$ locally uniformly by standard arguments. Moreover $\tilde{\gamma}$ is strictly convex, and even more, $\left\langle\nabla^{2} \tilde{\gamma}(p, \tau) \xi, \xi\right\rangle>0$ for $(p, \tau) \in \mathbb{R}^{n} \times(0, \infty)$ and $\xi \in \mathbb{R}^{n} \backslash\{0\}$. In fact, we obtain the strict convexity of $\tilde{\gamma}$ by the convexity of $\gamma$ since $G>0$. 
To see $\left\langle\nabla^{2} \tilde{\gamma}(p, \tau) \xi, \xi\right\rangle>0$ for $(p, \tau) \in \mathbb{R}^{n} \times(0, \infty)$ and $\xi \in \mathbb{R}^{n} \backslash\{0\}$, we define $\phi(p, \tau):=$ $\left\langle\nabla^{2} \tilde{\gamma}(p, \tau) \xi, \xi\right\rangle=\left(\gamma * \frac{\partial^{2} G}{\partial \xi^{2}}(\cdot, \tau)\right)(p)$. We shall assume that there exists $\left(p_{0}, \tau_{0}\right) \in \mathbb{R}^{n} \times(0, \infty)$ with $\phi\left(p_{0}, \tau_{0}\right)=0$ and derive a contradiction. We observe that $\phi$ is a solution of the heat equation and $\phi \geqslant 0$ in $\mathbb{R}^{n} \times(0, \infty)$. Using the strong maximum principle for the heat equation, we get $\phi \equiv 0$ for $\mathbb{R}^{n} \times\left(0, \tau_{0}\right]$. This implies that the function $\sigma \mapsto \tilde{\gamma}(p+\sigma \xi, \tau)$ is linear for $\tau \in\left(0, \tau_{0}\right]$, which contradicts the strict convexity of $\tilde{\gamma}$.

The sequence $\{\tilde{\gamma}(\cdot, \tau)\}$ gives an approximation of $\gamma$. However, unfortunately $\tilde{\gamma}(\cdot, \tau)$ is not positively homogeneous of degree 1 . By using $\tilde{\gamma}$, we shall produce a function which satisfies $(\gamma 1)-(\gamma 5)$ and approximates $\gamma$. We take $\bar{\tau}>0$ satisfying $0 \in \mathcal{F}_{\tau} \backslash \partial \mathcal{F}_{\tau}$ for $\tau \leqslant \bar{\tau}$, where $\mathcal{F}_{\tau}=\{p ; \tilde{\gamma}(p, \tau) \leqslant 1\}$. We define

$$
\gamma_{\tau}(p)=\inf \left\{r ; r>0, p / r \in \mathcal{F}_{\tau}\right\} .
$$

As we shall see later, $\gamma_{\tau}$ is as desired, i.e., it has properties $(\gamma 1)-(\gamma 5)$, there exists a uniform bound $\Lambda_{\gamma}$ in $(\gamma 3)$ for $\gamma_{\tau}$, and $\gamma_{\tau} \rightarrow \gamma$ as $\tau \rightarrow 0$ locally uniformly.

The homogeneity $(\gamma 2)$ and the convexity $(\gamma 4)$ easily follow from the definition of $\gamma_{\tau}$.

The smoothness $(\gamma 1)$ follows from an estimate of $D \tilde{\gamma}$ on $\partial \mathcal{F}_{\tau}$. Since $\tilde{\gamma}$ is strictly convex and $\tilde{\gamma}(0, \tau)<1$ for $\tau<\bar{\tau}$, we get $|D \tilde{\gamma}(p, \tau)| \neq 0$, in particular, $\langle D \tilde{\gamma}(p, \tau), p\rangle>0$ for $(p, \tau) \in$ $\partial \mathcal{F}_{\tau} \times(0, \bar{\tau})$. We define $g(r, q)=\tilde{\gamma}(r q, \tau)-1$ for $r>0$ and $q \in S^{n-1}$, and get

$$
\frac{\partial g}{\partial r}(r, q)=\frac{1}{r}\langle D \tilde{\gamma}(p, \tau), p\rangle>0
$$

for $p=r q \in \partial \mathcal{F}_{\tau}$. This implies that there exists a smooth function $\varphi=\varphi(q)$ for $q \in S^{n-1}$ with $g(\varphi(q), q)=0$ so that $\varphi(q) q \in \partial \mathcal{F}_{\tau}$ since $\partial \mathcal{F}_{\tau}=\{p ; \tilde{\gamma}(p, \tau)=1\}=\left\{p ; \gamma_{\tau}(p)=1\right\}$. This yields $\gamma_{\tau}(p)=|p|(\varphi(p /|p|))^{-1}$ so that $\gamma_{\tau}$ is smooth outside the origin.

Property $(\gamma 5)$ follows from the strict convexity of $\mathcal{F}_{\tau}$. In fact, these two conditions are equivalent (see [G2, Remark 1.7.5]). We indicate here the proof that the strict convexity of $\mathcal{F}_{\tau}$ implies the strict convexity of $\gamma_{\tau}^{2}$. By $(\gamma 4)$ we get $\left\{p ; \gamma_{\tau}(p) \leqslant c\right\}=\left\{c p ; \gamma_{\tau}(p) \leqslant 1\right\}=\{c p ; \tilde{\gamma}(p, \tau) \leqslant 1\}$ for $c>0$. This and $D^{2} \tilde{\gamma}>0$ yield

$$
\left\langle R_{\xi} D^{2} \gamma_{\tau}(p) R_{\xi} \eta, \eta\right\rangle>0 \quad \text { for } p, \eta \in \mathbb{R}^{n} \backslash\{0\} \text { with }\langle\xi, \eta\rangle=0,
$$

where $\xi=D \gamma_{\tau}(p)$ and $R_{\xi}=I-(\xi \otimes \xi) /|\xi|^{2}$. Since $R_{\xi} \eta=\eta$ we obtain

$$
\begin{aligned}
\left\langle R_{\xi} D^{2} \gamma_{\tau}(p) R_{\xi} \eta, \eta\right\rangle & =\left\langle D^{2} \gamma_{\tau}(p) \eta-|\xi|^{-2} \xi \otimes \xi D^{2} \gamma_{\tau}(p) \eta, \eta\right\rangle \\
& =\left\langle D^{2} \gamma_{\tau}(p) \eta, \eta\right\rangle-|\xi|^{-2}\left\langle\xi, D^{2} \gamma_{\tau}(p) \eta\right\rangle\langle\xi, \eta\rangle=\left\langle D^{2} \gamma_{\tau}(p) \eta, \eta\right\rangle,
\end{aligned}
$$

i.e., $\left\langle D^{2} \gamma_{\tau}(p) \eta, \eta\right\rangle>0$ for $p, \eta \in \mathbb{R}^{n} \backslash\{0\}$ with $\langle\xi, \eta\rangle=0$. By using this inequality we get $\operatorname{Ker} D^{2} \gamma_{\tau}(p)=\mathbb{R} p=\{c p ; c \in \mathbb{R}\}$ (see [G2, Remark 1.7.5]). In fact, we obtain $D^{2} \gamma_{\tau}(p) p=0$ since $\gamma_{\tau}$ is positively homogeneous of degree 1 . To see $\operatorname{Ker} D^{2} \gamma_{\tau}(p)=\mathbb{R} p$ we shall assume that there exists $q \in \operatorname{Ker} D^{2} \gamma_{\tau}(p)$ with $\langle p, q\rangle=0$ and derive a contradiction. We set $x:=-\langle\xi, q\rangle p+$ $\gamma_{\tau}(p) q=-\langle\xi, q\rangle p+\langle\xi, p\rangle q$. Then we obtain $x \neq 0,\langle\xi, x\rangle=0$ and $D^{2} \gamma_{\tau}(p) x=0$. However, $\left\langle D^{2} \gamma_{\tau}(p) x, x\right\rangle>0$ since $\langle\xi, x\rangle=0$. This is a contradiction.

For $x \neq 0$, we set $x=c_{1} p+c_{2} q$ for $p, q \in \mathbb{R}^{n}$ and $c_{1}, c_{2} \in \mathbb{R}$ with $\langle p, q\rangle=0$ and shall prove that $\left\langle D^{2} \gamma_{\tau}(p)^{2} x, x\right\rangle>0$. If $c_{2}=0$, then $\left\langle D^{2} \gamma_{\tau}(p)^{2} x, x\right\rangle=2 c_{1}^{2} \gamma_{\tau}(p)^{2}>0$. If $c_{2} \neq 0$, then $\left\langle D^{2} \gamma(p)^{2} x, x\right\rangle \geqslant c_{2}^{2}\left\langle D^{2} \gamma_{\tau}(p) q, q\right\rangle>0$ since $\operatorname{Ker} D^{2} \gamma_{\tau}(p)=\mathbb{R} p$. We have thus established $(\gamma 5)$.

The uniform bound $(\gamma 3)$ and the locally uniform convergence $\gamma_{\tau} \rightarrow \gamma$ as $\tau \rightarrow 0$ easily follow from the locally uniform convergence $\tilde{\gamma}(\cdot, \tau) \rightarrow \gamma$ as $\tau \rightarrow 0$ and the homogeneity of each function. 


\section{Properties of the anisotropic distance function}

In this section, we derive some properties of the anisotropic distance function, which follow from those of the support function. Most of them have already been proved in [BP2] for the support function and in [ElS2] for the anisotropic distance function. However, we need to refine some of them for our purpose. Especially, a refined version of an estimate of $\partial_{t} d$ is crucial for the proof of our uniform convergence result.

We list some properties of $\gamma^{\circ}$ :

$$
\begin{aligned}
& \gamma\left(D \gamma^{\circ}(p)\right)=\gamma^{\circ}(D \gamma(p))=1 \text { for } p \neq 0, \\
& \gamma(p) D \gamma^{\circ}(D \gamma(p))=\gamma^{\circ}(p) D \gamma\left(D \gamma^{\circ}(p)\right)=p \text { for } p \neq 0, \\
& D^{2} \gamma^{\circ}(p) D \gamma\left(D \gamma^{\circ}(p)\right)=D^{2} \gamma(p) D \gamma^{\circ}(D \gamma(p))=0 \text { for } p \neq 0 .
\end{aligned}
$$

We only give a few remarks on the proof; see [BP2] for the details.

Since $\left\{p \in \mathbb{R}^{n} ; \gamma(p) \leqslant 1\right\}$ is convex, we see that $\left(\gamma^{\circ}\right)^{\circ}=\gamma$ by convex analysis. The first equalities of (3.1)- 3.3) easily follow from this duality formula. Moreover, the identity of (3.3) follows from $(3.1)$ by differentiation. In [BP2], to prove (3.1), one needs to assume that, for $p \neq 0$, there exists a unique $q \in\left\{p \in \mathbb{R}^{n} ; \gamma(p) \leqslant 1\right\}$ satisfying $\gamma^{\circ}(p)=\langle p, q\rangle$. In our situation, this is the case since $\gamma^{2}$ is strictly convex.

\subsection{Properties of $d$}

We state the general properties of the anisotropic distance from a subset in $\mathbb{R}^{n}$.

LEMMA 3.1 Assume that $\gamma$ satisfies $(\gamma 1)-(\gamma 5)$. Let $\Gamma \subset \mathbb{R}^{n}$ be a closed subset. Define $d(x)=$ $\Xi(x, \Gamma)$. Then $d$ is a viscosity supersolution of

$$
\left.\begin{array}{c}
\gamma(\nabla d)=1, \quad-\gamma(\nabla d)=-1 \\
-\left\langle\nabla^{2} d D \gamma(\nabla d), D \gamma(\nabla d)\right\rangle=0
\end{array}\right\} \quad \text { in }\left\{x \in \mathbb{R}^{n} ; d(x)>0\right\} .
$$

Lemma 3.1 comes from 3.1 and the derivative of (3.1) in the direction $D \gamma(\nabla d)$. Fortunately, however, we can prove the first equation without the differentiability of $d$ by using viscosity notions. We shall give the proof for completeness. In the theory of viscosity solutions, we often consider the upper and lower semicontinuous envelopes of functions to show that they are a viscosity subsolution and supersolution of an equation, respectively. However, since

$$
-\gamma^{\circ}(y-x) \leqslant d(x)-d(y) \leqslant \gamma^{\circ}(x-y) \quad \text { for all } x, y \in \mathbb{R}^{n},
$$

$d$ is Lipschitz continuous. Therefore we do not have to consider a lower semicontinuous envelope.

Proof of Lemma 3.1. Fix $x_{0} \in\{x ; d(x)>0\}$. Let $\varphi \in C^{2}\left(\mathbb{R}^{n}\right)$ satisfy

$$
d(x)-\varphi(x) \geqslant d\left(x_{0}\right)-\varphi\left(x_{0}\right) \quad \text { for } x \in \mathbb{R}^{n} .
$$

Since $\Gamma$ is closed, there exists $y_{0} \in \Gamma$ satisfying

$$
d\left(x_{0}\right)=\gamma^{\circ}\left(x_{0}-y_{0}\right) .
$$

Then

$$
\gamma^{\circ}\left(x-y_{0}\right)-\varphi(x) \geqslant d(x)-\varphi(x) \geqslant d\left(x_{0}\right)-\varphi\left(x_{0}\right)=\gamma^{\circ}\left(x_{0}-y_{0}\right)-\varphi\left(x_{0}\right) .
$$


We first show the first equation of the lemma. Since $\gamma$ is strictly convex, for every $x$ there exists a unique vector $q_{x} \in\{p ; \gamma(p) \leqslant 1\}$ satisfying

$$
\gamma\left(x-y_{0}\right)=\left\langle x-y_{0}, q_{x}\right\rangle \text { and } \gamma\left(q_{x}\right)=1 .
$$

We set $q_{0}=q_{x_{0}}$. Then $q_{x} \rightarrow q_{0}$ as $x \rightarrow x_{0}$ by taking a subsequence of $\left\{q_{x}\right\}$ if necessary. By a calculation we find that

$$
\left\langle x-y_{0}, q_{x}\right\rangle-\varphi(x)=\gamma^{\circ}\left(x-y_{0}\right)-\varphi(x) \geqslant \gamma^{\circ}\left(x_{0}-y_{0}\right)-\varphi\left(x_{0}\right) \geqslant\left\langle x_{0}-y_{0}, q_{x}\right\rangle-\varphi(x) .
$$

Thus

$$
\left\langle x-x_{0}, q_{x}\right\rangle \geqslant \varphi(x)-\varphi\left(x_{0}\right)=\left\langle x-x_{0}, \nabla \varphi\left(x_{0}\right)\right\rangle+o\left(\left|x-x_{0}\right|\right)
$$

as $\left|x-x_{0}\right| \rightarrow 0$. We now divide by $\left|x-x_{0}\right|$ and let $x \rightarrow x_{0}$ to find, for $e \in S^{n-1}$,

$$
\left\langle e, q_{0}-\nabla \varphi\left(x_{0}\right)\right\rangle \geqslant 0 .
$$

Thus $q_{0}=\nabla \varphi\left(x_{0}\right)$. From $\gamma\left(q_{0}\right)=1$, we obtain

$$
\gamma\left(\nabla \varphi\left(x_{0}\right)\right) \geqslant 1 \quad \text { and } \quad-\gamma\left(\nabla \varphi\left(x_{0}\right)\right) \geqslant-1 .
$$

Next we show the second equation. We differentiate 3.1 in the direction $D \gamma\left(D \gamma^{\circ}(p)\right)$ to get

$$
\left\langle D^{2} \gamma^{\circ}(p) D \gamma\left(D \gamma^{\circ}(p)\right), D \gamma\left(D \gamma^{\circ}(p)\right)\right\rangle=0 \quad \text { for } p \neq 0 \text {. }
$$

Here $x_{0} \in\{x ; d(x)>0\}$ implies $x_{0} \neq y_{0}$. We can now calculate the derivatives of $\gamma^{\circ}\left(\cdot-y_{0}\right)$ at $x_{0}$ and obtain

$$
D \gamma^{\circ}\left(x_{0}-y_{0}\right)=\nabla \varphi\left(x_{0}\right), \quad D^{2} \gamma^{\circ}\left(x_{0}-y_{0}\right) \geqslant \nabla \varphi\left(x_{0}\right) \text {. }
$$

We thus get

$$
\begin{aligned}
-\left\langle\nabla^{2} \varphi\left(x_{0}\right) D \gamma\left(\nabla \varphi\left(x_{0}\right)\right)\right. & \left., D \gamma\left(\nabla \varphi\left(x_{0}\right)\right)\right\rangle \\
& \geqslant\left\langle D^{2} \gamma^{\circ}\left(x_{0}-y_{0}\right) D \gamma\left(D \gamma^{\circ}\left(x_{0}-y_{0}\right)\right), D \gamma\left(D \gamma^{\circ}\left(x_{0}-y_{0}\right)\right)\right\rangle=0,
\end{aligned}
$$

which yields the second equation.

We remark that for $d(x)=\Xi(\Gamma, x)$, we obtain a similar result. However, the sign of $\nabla d$ is reversed, i.e., the reversed version of the distance is a viscosity supersolution of $\gamma(-\nabla d)=1,-\gamma(-\nabla d)=$ -1 , and $-\left\langle\nabla^{2} d D \gamma(-\nabla d), D \gamma(-\nabla d)\right\rangle=0$. Note that we do not treat the reversed version of the distance in this paper.

We next obtain properties of anisotropic distance functions from the moving interface $\Gamma_{t}$.

LEMMA 3.2 Assume that $\beta, \gamma$ and $f$ satisfy $(\beta 1)-(\beta 3),(\gamma 1)-(\gamma 5)$, and $(f 1)$, respectively. Let $u$ be a viscosity solution of (2.4) with initial data $u(x, 0)=d_{0}(x)$. Let $d(x, t)$ be an anisotropic distance function defined by

$$
d(x, t)= \begin{cases}\Xi\left(x, \Gamma_{t}\right) & \text { for } x \in\{u(x, t) \geqslant 0\}, \\ -\Xi\left(x, \Gamma_{t}\right) & \text { for } x \in\{u(x, t)<0\},\end{cases}
$$

where $\Gamma_{t}=\left\{x \in \mathbb{R}^{n} ; u(x, t)=0\right\}$. Then $d$ is a viscosity supersolution of 2.4 in $\{(x, t) \in$ $\left.\mathbb{R}^{n} \times(0, T) ; d(x, t)>0\right\}$.

This lemma is already proved in [ElS2, Lemma 3.3]. Their lemma has an error term $C\left(\Lambda_{\gamma}\right)|\nabla d| d$. However this term disappears if $f$ is independent of the space variable $x$. 


\subsection{Estimate of $\partial_{t} d$}

In this section we prepare an estimate of $\partial_{t} d$ which is useful to construct our supersolution.

LEMMA 3.3 Assume that $\beta, \gamma$ and $f$ satisfy $(\beta 1)-(\beta 3),(\gamma 1)-(\gamma 5)$ and $(f 1)$, respectively. Let $d$ be the anisotropic distance function defined in Lemma 3.2 .

(i) Let $\mu$ be the function defined by $\mu(\sigma)=\int_{0}^{\sigma} s /(1+s) \mathrm{d} s$. Then

$$
\mu(d(\hat{x}, t)) \geqslant \mu(d(\hat{x}, \hat{t}))-L_{\beta, f}(t-\hat{t})
$$

for $(\hat{x}, t),(\hat{x}, \hat{t}) \in\{(x, t) ; d(x, t)>0\}$ provided that $0 \leqslant \hat{t} \leqslant t<T$, where $L_{\beta, f}$ is a positive constant depends only on $n, \Lambda_{\beta}$, and $\Lambda_{f}$.

(ii) The anisotropic distance function $d$ is a viscosity supersolution of

$$
\partial_{t} d=-L_{\beta, f}\left(1+\frac{1}{d}\right) \quad \text { in }\{(x, t) ; d(x, t)>0\} .
$$

This lemma is a refined version of that in [ElS2]. In particular, the constant $L_{\beta, f}$ is independent of any derivatives of $\gamma$. This is the main advantage over [ElS2] so that we obtain our uniform convergence result.

Proof. Fix $(\hat{x}, \hat{t}) \in\{(x, t) ; d(x, t)>0\}$ and let $\hat{r}=d(\hat{x}, \hat{t})$. We define the function $z: \mathbb{R}^{n} \times$ $[0, T) \rightarrow \mathbb{R}$ by

$$
z(x, t)=\mu(\hat{r})-L(t-\hat{t})-\mu\left(\gamma^{\circ}(\hat{x}-x)\right),
$$

where $L$ is a positive constant to be determined later. Since $\gamma^{\circ} \in C^{2}\left(\mathbb{R}^{n} \backslash\{0\}\right)$ we observe that $z \in C^{2,1}\left(\left(\mathbb{R}^{n} \backslash\{\hat{x}\}\right) \times[0, T)\right) \cap C^{1,1}\left(\mathbb{R}^{n} \times[0, T)\right)$. By a straightforward calculation we obtain

$$
\begin{aligned}
\partial_{t} z(x, t) & =-L, \\
\nabla z(x, t) & =\mu^{\prime}\left(\gamma^{\circ}(\hat{q})\right) D \gamma^{\circ}(\hat{q}), \\
\nabla^{2} z(x, t) & =-\mu^{\prime \prime}\left(\gamma^{\circ}(\hat{q})\right) D \gamma^{\circ}(\hat{q}) \otimes D \gamma^{\circ}(\hat{q})-\mu^{\prime}\left(\gamma^{\circ}(\hat{q})\right) D^{2} \gamma^{\circ}(\hat{q})
\end{aligned}
$$

for $x \neq \hat{x}$, where $\hat{q}=\hat{x}-x$. We observe that $z \in C^{1,1}\left(\mathbb{R}^{n} \times[0, T)\right)$ and $\nabla z(\hat{x}, t)=0$.

In the following argument, we shall verify that $z$ is a viscosity subsolution of (2.4). For this purpose, we give an estimate of the second term of $(2.4)$ for $z$ provided that $\hat{x}-x \neq 0$. First we remark that $\mu^{\prime}>0$ on $\mathbb{R}$. Then we obtain

$$
\begin{aligned}
\gamma(\nabla z) & =\mu^{\prime}\left(\gamma^{\circ}(\hat{q})\right) \gamma\left(D \gamma^{\circ}(\hat{q})\right)=\mu^{\prime}\left(\gamma^{\circ}(\hat{q})\right), \\
D \gamma(\nabla z) & =D \gamma\left(D \gamma^{\circ}(\hat{q})\right), \\
D^{2} \gamma(\nabla z) & =\frac{1}{\mu^{\prime}\left(\gamma^{\circ}(\hat{q})\right)} D^{2} \gamma\left(D \gamma^{\circ}(\hat{q})\right) .
\end{aligned}
$$

Therefore, by straightforward calculation,

$$
\begin{aligned}
\operatorname{tr}\left\{\gamma(\nabla z) D^{2} \gamma(\nabla z) \nabla^{2} z\right\}= & -\mu^{\prime \prime}\left(\gamma^{\circ}(\hat{q})\right)\left\langle D^{2} \gamma\left(D \gamma^{\circ}(\hat{q})\right) D \gamma^{\circ}(\hat{q}), D \gamma^{\circ}(\hat{q})\right\rangle \\
& +\mu^{\prime}\left(\gamma^{\circ}(\hat{q})\right) \operatorname{div}\left\{D \gamma\left(D \gamma^{\circ}(\hat{q})\right)\right\} .
\end{aligned}
$$

By calculating the derivative of the second equality of [3.1, we obtain

$$
D^{2} \gamma(p) D \gamma^{\circ}(D \gamma(p))=0 \quad \text { for } p \neq 0 \text {. }
$$


Taking $p=D \gamma^{\circ}(\hat{q})$ yields

$$
D^{2} \gamma\left(D \gamma^{\circ}(\hat{q})\right) D \gamma^{\circ}\left(D \gamma\left(D \gamma^{\circ}(\hat{q})\right)\right)=0
$$

By (3.2) and since $D \gamma^{\circ}$ is positively homogeneous of degree 0 , we obtain

$$
D^{2} \gamma\left(D \gamma^{\circ}(\hat{q})\right) D \gamma^{\circ}(\hat{q})=0
$$

i.e., the first term on the right hand side of (3.4) vanishes. Moreover, from (3.2) and since $\gamma^{\circ}$ is positively homogeneous of degree 1 , we obtain

$$
\operatorname{div} D \gamma\left(D \gamma^{\circ}(p)\right)=\operatorname{div}\left(\frac{p}{\gamma^{\circ}(p)}\right)=\frac{n \gamma^{\circ}(p)-\left\langle p, D \gamma^{\circ}(p)\right\rangle}{\gamma^{\circ}(p)^{2}}=\frac{n-1}{\gamma^{\circ}(p)}
$$

Combining these and $\mu^{\prime}(\sigma)=\sigma /(1+\sigma)$ gives

$$
\operatorname{tr}\left\{\gamma(\nabla z) D^{2} \gamma(\nabla z) \nabla^{2} z\right\}=-\mu^{\prime}\left(\gamma^{\circ}(\hat{q})\right) \frac{n-1}{\gamma^{\circ}(\hat{q})}=-\frac{n-1}{1+\gamma^{\circ}(\hat{q})} \geqslant-(n-1) .
$$

We are now in a position to verify that $z$ is a viscosity subsolution of (2.4). First we verify this in $\left(\mathbb{R}^{n} \backslash\{\hat{x}\}\right) \times(0, T)$. By 3.5 and since $\mu^{\prime}<1$ we obtain

$$
\beta(\nabla z) \partial_{t} z-\operatorname{tr}\left\{\gamma(\nabla z) D^{2} \gamma(\nabla z) \nabla^{2} z\right\}-\gamma(\nabla z) f \leqslant-\frac{L}{\Lambda_{\beta}}+n-1+|f| .
$$

We take $L>0$ satisfying $-L / \Lambda_{\beta}+n-1+|f| \leqslant 0$, which shows that $z$ is a viscosity subsolution of 2.4$)$ in $\left(\mathbb{R}^{n} \backslash\{\hat{x}\}\right) \times(0, T)$. Here we take $L=\Lambda_{\beta}\left(n+\Lambda_{f}\right)=: L_{\beta, f}$.

Next we verify that $z$ is a viscosity subsolution in a neighborhood of $\{\hat{x}\} \times(0, T)$. Let $\hat{s} \in(0, T)$ and let $\varphi \in C^{2}\left(\mathbb{R}^{n} \times(0, T)\right)$ satisfy

$$
z(x, t)-\varphi(x, t)<z(\hat{x}, \hat{s})-\varphi(\hat{x}, \hat{s}) \quad \text { for }(x, t) \in \mathbb{R}^{n} \times(0, T) \backslash\{(\hat{x}, \hat{s})\} .
$$

Then $\nabla \varphi(\hat{x}, \hat{s})=\nabla z(\hat{x}, \hat{s})=0$. Fix $e \in S^{n-1}$ and define

$$
\varphi_{\tau}(x, t)=\varphi(x, t)+\tau\langle e, x\rangle .
$$

Then, for sufficiently small $\tau>0$, there exists $x_{\tau} \in\{x ; d(x, \hat{s})>0\}$ satisfying

$$
\begin{aligned}
& z(\cdot, \hat{s})-\varphi_{\tau}(\cdot, \hat{s}) \leqslant z\left(x_{\tau}, \hat{s}\right)-\varphi_{\tau}\left(x_{\tau}, \hat{s}\right) \quad \text { in some neighborhood of } \hat{x}, \\
& x_{\tau} \neq \hat{x} \text { and } x_{\tau} \rightarrow \hat{x} \text { as } \tau \rightarrow 0 .
\end{aligned}
$$

We only verify that $x_{\tau} \neq \hat{x}$. If $x_{\tau}=\hat{x}$, then $\nabla \varphi_{\tau}\left(x_{\tau}, \hat{s}\right)=\nabla \varphi(\hat{x}, \hat{s})+\tau e=\tau e \neq 0$. However, also $\nabla \varphi_{\tau}\left(x_{\tau}, \hat{s}\right)=\nabla z\left(x_{\tau}, \hat{s}\right)=\nabla z(\hat{x}, \hat{s})=0$. This is a contradiction.

We now observe that

$$
\left.\begin{array}{rl}
\partial_{t} z\left(x_{\tau}, \hat{s}\right) & \rightarrow \partial_{t} z(\hat{x}, \hat{s})=\partial_{t} \varphi(\hat{x}, \hat{s}), \\
\nabla \varphi_{\tau}\left(x_{\tau}, \hat{s}\right) & \rightarrow \nabla \varphi(\hat{x}, \hat{s}), \\
\nabla^{2} \varphi_{\tau}\left(x_{\tau}, \hat{s}\right) & \rightarrow \nabla^{2} \varphi(\hat{x}, \hat{s})
\end{array}\right\} \quad \text { as } \tau \rightarrow 0 .
$$


Moreover, $\nabla \varphi_{\tau}\left(x_{\tau}, \hat{s}\right)=\nabla z\left(x_{\tau}, \hat{s}\right)$ and $\nabla^{2} \varphi_{\tau}\left(x_{\tau}, \hat{s}\right) \geqslant \nabla^{2} z\left(x_{\tau}, \hat{s}\right)$ since $z-\varphi_{\tau}(\cdot, \hat{s})$ attains its maximum at $\hat{x}$. Therefore

$$
\begin{aligned}
& {\left[\beta(\nabla \varphi) \partial_{t} \varphi-\operatorname{tr}\left\{\gamma(\nabla \varphi) D^{2} \gamma(\nabla \varphi) \nabla^{2} \varphi\right\}-\gamma(\nabla \varphi) f\right]_{*}(\hat{x}, \hat{s}) } \\
& \leqslant \lim _{\tau \rightarrow 0}\left[\beta\left(\nabla \varphi_{\tau}\right) \partial_{t} z-\operatorname{tr}\left\{\gamma\left(\nabla \varphi_{\tau}\right) D^{2} \gamma\left(\nabla \varphi_{\tau}\right) \nabla^{2} \varphi_{\tau}\right\}-\gamma\left(\nabla \varphi_{\tau}\right) f\right]\left(x_{\tau}, \hat{s}\right) \\
& \leqslant \lim _{\tau \rightarrow 0}\left[\beta(\nabla z) \partial_{t} z-\operatorname{tr}\left\{\gamma(\nabla z) D^{2} \gamma(\nabla z) \nabla^{2} z\right\}-\gamma(\nabla z) f\right]\left(x_{\tau}, \hat{s}\right) \leqslant 0 .
\end{aligned}
$$

We conclude that $z$ is a viscosity subsolution of $(2.4)$ in $\mathbb{R}^{n} \times(0, T)$.

We now verify (i1). From [CGG1], we know that $u_{k}^{+}(x, t):=\min (k \max (u(x, t), 0), 1)$ is a viscosity solution of (2.4) for $k>0$ since $u(x, t)$ is a viscosity solution of (2.4). Moreover, $u^{\infty}(x, t):=\chi_{\{u>0\}}(x, t)=\lim _{k \rightarrow \infty} \inf \left\{u_{j}^{+}(y, s) ;|y-x|+|s-t|<1 / k, j>k\right\}$, is a viscosity supersolution of (2.4) (see [CIL, Lemma 6.1]). We now consider the set $U=\left\{x ; \gamma^{\circ}(\hat{x}-x)<\hat{r}\right\}$. Then we obtain

$$
z(x, \hat{t})=\mu(\hat{r})-\mu\left(\gamma^{\circ}(\hat{x}-x)\right) \leqslant \mu(\hat{r})=\mu(\hat{r}) u^{\infty}(x, \hat{t}) \quad \text { for } x \in U
$$

since $\mu>0$ and $d(x, \hat{t})>0$ for $x \in U$. Moreover, for $(x, t) \in \partial U \times[\hat{t}, T)$,

$$
z(x, t)=-L_{\beta, f}(t-\hat{t}) \leqslant 0 \leqslant \mu(\hat{r}) u^{\infty}(x, t) .
$$

Therefore, by the comparison principle,

$$
z(x, t) \leqslant \mu(\hat{r}) u^{\infty}(x, t) \quad \text { for }(x, t) \in \bar{U} \times[\hat{t}, T) .
$$

For $t \in[\hat{t}, T)$, fix $\hat{y} \in \mathbb{R}^{n}$ satisfying $u(\hat{y}, t)=0$ and $d(\hat{x}, t)=\gamma^{\circ}(\hat{x}-\hat{y})$. If $\hat{y} \in \bar{U}$, then

$$
\begin{aligned}
0=\mu(\hat{r}) u^{\infty}(\hat{y}, t) & \geqslant z(\hat{y}, t)=\mu(\hat{r})-L_{\beta, f}(t-\hat{t})-\mu\left(\gamma^{\circ}(\hat{x}-\hat{y})\right) \\
& =\mu(d(\hat{x}, \hat{t}))-L_{\beta, f}(t-\hat{t})-\mu(d(\hat{x}, t)),
\end{aligned}
$$

or

$$
\mu(d(\hat{x}, t)) \geqslant \mu(d(\hat{x}, \hat{t}))-L_{\beta, f}(t-\hat{t})
$$

If $\hat{y} \notin \bar{U}$, then

$$
\mu(d(\hat{x}, t))=\mu\left(\gamma^{\circ}(\hat{x}-\hat{y})\right) \geqslant \mu(\hat{r})=\mu(d(\hat{x}, \hat{t})) \geqslant \mu(d(\hat{x}, \hat{t}))-L_{\beta, f}(t-\hat{t}),
$$

which yields (i).

Finally, we verify (iii). Let $\left(x_{0}, t_{0}\right) \in\{(x, t) ; d(x, t)>0\}$ and let $\varphi \in C^{2}\left(\mathbb{R}^{n} \times(0, T)\right)$ satisfy

$$
d(x, t)-\varphi(x, t) \geqslant d\left(x_{0}, t_{0}\right)-\varphi\left(x_{0}, t_{0}\right)=0 \quad \text { for }(x, t) \in \mathbb{R}^{n} \times(0, T) .
$$

From (ii) we observe that $d(x, t)$ is left continuous in time in the sense that

$$
\lim _{x \rightarrow x_{0}} \lim _{t \uparrow t_{0}} d(x, t)=d\left(x_{0}, t_{0}\right)
$$

(see [EIS2, Proposition 3.5]). Then there exists a constant $r>0$ satisfying

$$
d\left(x_{0}, t\right)>0 \quad \text { for } t \in\left(t_{0}-r, t_{0}\right] .
$$


By using (ii) we see that

$$
\mu\left(\varphi\left(x_{0}, t_{0}\right)\right)=\mu\left(d\left(x_{0}, t_{0}\right)\right) \geqslant \mu\left(d\left(x_{0}, t\right)\right)-L_{\beta, f}\left(t_{0}-t\right) \geqslant \mu\left(\varphi\left(x_{0}, t\right)\right)-L_{\beta, f}\left(t_{0}-t\right),
$$

or

$$
\frac{\mu\left(\varphi\left(x_{0}, t_{0}\right)\right)-\mu\left(\varphi\left(x_{0}, t\right)\right)}{t_{0}-t} \geqslant-L_{\beta, f}
$$

Letting $t \rightarrow t_{0}$ yields

$$
\mu^{\prime}\left(\varphi\left(x_{0}, t_{0}\right)\right) \partial_{t} \varphi\left(x_{0}, t_{0}\right) \geqslant-L_{\beta, f} .
$$

Since $\varphi\left(x_{0}, t_{0}\right)=d\left(x_{0}, t_{0}\right)$ and $\mu^{\prime}(\sigma)=\sigma /(1+\sigma)$ we obtain

$$
\partial_{t} \varphi\left(x_{0}, t_{0}\right) \geqslant-L_{\beta, f}\left(1+\frac{1}{d\left(x_{0}, t_{0}\right)}\right) .
$$

\subsection{Truncated anisotropic distance function}

In this section we shall prepare several estimates of truncated anisotropic distance functions to construct our supersolution having a uniform estimate.

We first recall a function $\eta$ introduced in [ESS]. We fix $\delta \in(0,1)$ and we consider a function $\eta \in C^{\infty}(\mathbb{R})$ satisfying

$$
\begin{aligned}
& \eta(\sigma)= \begin{cases}\sigma-\delta & \text { if } \sigma \geqslant \delta / 2, \\
-\delta & \text { if } \sigma \leqslant \delta / 4,\end{cases} \\
& 0 \leqslant \eta^{\prime} \leqslant C_{\eta}, \quad\left|\eta^{\prime \prime}\right| \leqslant C_{\eta} / \delta,
\end{aligned}
$$

where $C_{\eta}$ is a constant independent of $\sigma$ and $\delta$.

We now introduce a truncated anisotropic distance function. Let $u$ be a viscosity solution of 2.4 with initial data $u_{0}(x)=d_{0}(x)$, where $d_{0}$ is the anisotropic signed distance function defined by 2.13 . We set

$$
\begin{aligned}
O_{t} & =\left\{x \in \mathbb{R}^{n} ; u(x, t)>0\right\}, \\
D_{t} & =\left\{x \in \mathbb{R}^{n} ; u(x, t)<0\right\}, \\
\Gamma_{t} & =\left\{x \in \mathbb{R}^{n} ; u(x, t)=0\right\} .
\end{aligned}
$$

We now define the anisotropic signed distance function $d: \mathbb{R}^{n} \times[0, T) \rightarrow \mathbb{R}$ from the moving interface $\Gamma_{t}$ by

$$
d(x, t)= \begin{cases}\Xi\left(x, \Gamma_{t}\right) & \text { if } x \in O_{t} \cup \Gamma_{t}, \\ -\Xi\left(x, \Gamma_{t}\right) & \text { if } x \in D_{t}\end{cases}
$$

The truncated anisotropic distance function $\omega: \mathbb{R}^{n} \times[0, T) \rightarrow \mathbb{R}$ is defined by

$$
\omega(x, t)=\eta(d(x, t)) .
$$

Now we state some properties of $\omega$. 
Lemma 3.4 Assume that $\beta, \gamma$ and $f$ satisfy $(\beta 1)-(\beta 3),(\gamma 1)-(\gamma 5)$ and $(f 1)$, respectively. Then the truncated anisotropic distance function $\omega(x, t)=\eta(d(x, t))$ is a viscosity supersolution of

$$
\left.\begin{array}{rl}
\beta(\nabla \omega) \partial_{t} \omega-\operatorname{tr}\left\{D^{2} \alpha(\nabla \omega) \nabla^{2} \omega\right\}-\gamma(\nabla \omega) f & =-C_{\eta} / \delta \\
-|\nabla \omega| & =-C_{\gamma}
\end{array}\right\} \quad \text { in } \mathbb{R}^{n} \times(0, T),
$$

where $C_{\gamma}$ is a positive constant depending only on $\Lambda_{\gamma}$. Moreover, $\omega$ is a viscosity supersolution of

$$
\left.\begin{array}{rl}
\beta(\nabla \omega) \partial_{t} \omega-\operatorname{tr}\left\{D^{2} \alpha(\nabla \omega) \nabla^{2} \omega\right\}-\gamma(\nabla \omega) f & =0 \\
\pm \gamma(\nabla \omega) & = \pm 1
\end{array}\right\} \quad \text { in }\{(x, t) ; d(x, t)>\delta / 2\} .
$$

We remark that the equation in Lemma 3.4 is different from (2.4). We replace the second term of (2.4) by that of the Allen-Cahn equation (2.6) to get 3.8) or 3.9). This modification is useful to construct a supersolution for 2.97.

Proof. Let $\left(x_{0}, t_{0}\right) \in \mathbb{R}^{n} \times(0, T)$ and let $\varphi \in C^{2}\left(\mathbb{R}^{n} \times(0, T)\right)$ satisfy

$$
\omega(x, t)-\varphi(x, t)>\omega\left(x_{0}, t_{0}\right)-\varphi\left(x_{0}, t_{0}\right) \quad \text { whenever }(x, t) \neq\left(x_{0}, t_{0}\right) .
$$

We divide the argument into two cases: $d\left(x_{0}, t_{0}\right)>0$ and $d\left(x_{0}, t_{0}\right) \leqslant 0$.

Case 1. Assume that $d\left(x_{0}, t_{0}\right)>0$. Let $\tau \in(0,1)$ be a small parameter. We introduce a function $\eta_{\tau}$ approximating $\eta$, defined by

$$
\eta_{\tau}(\sigma)=\eta(\sigma)+\tau \sigma
$$

Then there exists a neighborhood $U=U\left(x_{0}, t_{0}\right)$ of $\left(x_{0}, t_{0}\right)$ and $\left(x_{\tau}, t_{\tau}\right) \in U$ satisfying

$$
\begin{gathered}
\eta_{\tau}(d(x, t))-\varphi(x, t) \geqslant \eta_{\tau}\left(d\left(x_{\tau}, t_{\tau}\right)\right)-\varphi\left(x_{\tau}, t_{\tau}\right) \quad \text { for }(x, t) \in U, \\
\left(x_{\tau}, t_{\tau}\right) \rightarrow\left(x_{0}, t_{0}\right) \quad \text { as } \tau \rightarrow 0 .
\end{gathered}
$$

Since $\eta_{\tau}^{\prime}=\eta^{\prime}+\tau \geqslant \tau>0$, there exists $\rho_{\tau}=\left(\eta_{\tau}\right)^{-1}$ and $\rho_{\tau}^{\prime}>0$. Define

$$
\bar{\varphi}_{\tau}(x, t)=\rho_{\tau}\left(\varphi(x, t)-\varphi\left(x_{\tau}, t_{\tau}\right)+\eta_{\tau}\left(d\left(x_{\tau}, t_{\tau}\right)\right)\right) .
$$

Then

$$
d(x, t)-\bar{\varphi}_{\tau}(x, t) \geqslant d\left(x_{\tau}, t_{\tau}\right)-\bar{\varphi}_{\tau}\left(x_{\tau}, t_{\tau}\right)=0 \quad \text { for }(x, t) \in U .
$$

By straightforward calculation we obtain

$$
\begin{aligned}
\partial_{t} \bar{\varphi}_{\tau} & =\rho_{\tau}^{\prime}(\kappa) \partial_{t} \varphi, \\
\nabla \bar{\varphi}_{\tau} & =\rho_{\tau}^{\prime}(\kappa) \nabla \varphi, \\
\nabla^{2} \bar{\varphi}_{\tau} & =\rho_{\tau}^{\prime \prime}(\kappa) \nabla \varphi \otimes \nabla \varphi+\rho^{\prime}(\kappa) \nabla^{2} \varphi,
\end{aligned}
$$

where $\kappa=\kappa(x, t)=\varphi(x, t)-\varphi\left(x_{\tau}, t_{\tau}\right)+\eta_{\tau}\left(d\left(x_{\tau}, t_{\tau}\right)\right)$. 
By Lemmas 3.1 and 3.2 .

$$
\left.\begin{array}{r}
\gamma\left(\nabla \bar{\varphi}_{\tau}\right)=1, \text { in particular, } \nabla \bar{\varphi}_{\tau} \neq 0 \\
\beta\left(\nabla \bar{\varphi}_{\tau}\right) \partial_{t} \bar{\varphi}_{\tau}-\operatorname{tr}\left\{\gamma\left(\nabla \bar{\varphi}_{\tau}\right) D^{2} \gamma\left(\nabla \bar{\varphi}_{\tau}\right) \nabla^{2} \bar{\varphi}_{\tau}\right\}-\gamma\left(\nabla \bar{\varphi}_{\tau}\right) f \geqslant 0
\end{array}\right\} \quad \text { at }\left(x_{\tau}, t_{\tau}\right),
$$

where we do not need to consider the place where the gradient of the unknown of 3.13 equals zero since $\nabla \bar{\varphi}_{\tau} \neq 0$ at $\left(x_{\tau}, t_{\tau}\right)$. By calculating the term including trace and using Lemma 3.1, we obtain

$$
\begin{aligned}
\operatorname{tr}\left\{\gamma\left(\nabla \bar{\varphi}_{\tau}\right) D^{2} \gamma\left(\nabla \bar{\varphi}_{\tau}\right) \nabla^{2} \bar{\varphi}_{\tau}\right\} & =\operatorname{tr}\left\{D^{2} \alpha\left(\nabla \bar{\varphi}_{\tau}\right) \nabla^{2} \bar{\varphi}_{\tau}\right\}-\left\langle\nabla^{2} \bar{\varphi}_{\tau} D \gamma\left(\nabla \bar{\varphi}_{\tau}\right), D \gamma\left(\nabla \bar{\varphi}_{\tau}\right)\right\rangle \\
& \geqslant \operatorname{tr}\left\{D^{2} \alpha\left(\nabla \bar{\varphi}_{\tau}\right) \nabla^{2} \bar{\varphi}_{\tau}\right\} .
\end{aligned}
$$

From 3.13 it now follows that

$$
\beta\left(\nabla \bar{\varphi}_{\tau}\right) \partial_{t} \bar{\varphi}_{\tau}-\operatorname{tr}\left\{D^{2} \alpha\left(\nabla \bar{\varphi}_{\tau}\right) \nabla^{2} \bar{\varphi}_{\tau}\right\}-\gamma\left(\nabla \bar{\varphi}_{\tau}\right) f \geqslant 0 \quad \text { at }\left(x_{\tau}, t_{\tau}\right) .
$$

By the homogeneity of $\beta, \gamma$ and $\alpha$ we obtain

$$
\beta\left(\nabla \bar{\varphi}_{\tau}\right)=\beta(\nabla \varphi), \quad \gamma\left(\nabla \bar{\varphi}_{\tau}\right)=\rho_{\tau}^{\prime}(\kappa) \gamma(\nabla \varphi), \quad D^{2} \alpha\left(\nabla \bar{\varphi}_{\tau}\right)=D^{2} \alpha(\nabla \varphi) .
$$

Combining 3.10 - 3.12 and the above, we deduce from 3.14 that

$$
\beta(\nabla \varphi) \partial_{t} \varphi-\operatorname{tr}\left\{D^{2} \alpha(\nabla \varphi) \nabla^{2} \varphi\right\}-\gamma(\nabla \varphi) f \geqslant \frac{\rho_{\tau}^{\prime \prime}(\kappa)}{\rho_{\tau}^{\prime}(\kappa)} \operatorname{tr}\left\{D^{2} \alpha(\nabla \varphi) \nabla \varphi \otimes \nabla \varphi\right\} \quad \text { at }\left(x_{\tau}, t_{\tau}\right) .
$$

Since $\alpha$ is positively homogeneous of degree 2 , we obtain

$$
\operatorname{tr}\left\{D^{2} \alpha(\nabla \varphi) \nabla \varphi \otimes \nabla \varphi\right\}=\left\langle D^{2} \alpha(\nabla \varphi) \nabla \varphi, \nabla \varphi\right\rangle=2 \alpha(\nabla \varphi)=\gamma(\nabla \varphi)^{2} .
$$

Moreover, $\rho_{\tau}^{\prime \prime}(\kappa) / \rho_{\tau}^{\prime}(\kappa)=-\eta_{\tau}^{\prime \prime}\left(\rho_{\tau}(\kappa)\right) \rho_{\tau}^{\prime}(\kappa)^{2}=-\eta_{\tau}^{\prime \prime}\left(\bar{\varphi}_{\tau}\right) \rho_{\tau}^{\prime}(\kappa)^{2}$. Hence

$$
\begin{aligned}
\frac{\rho_{\tau}^{\prime \prime}(\kappa)}{\rho_{\tau}^{\prime}(\kappa)} \operatorname{tr}\left\{D^{2} \alpha(\nabla \varphi) \nabla \varphi \otimes \nabla \varphi\right\} & =-\eta_{\tau}^{\prime \prime}\left(\bar{\varphi}_{\tau}\right)\left(\rho_{\tau}^{\prime}(\kappa) \gamma(\nabla \varphi)\right)^{2} \\
& =-\eta_{\tau}^{\prime \prime}\left(\bar{\varphi}_{\tau}\right) \gamma\left(\nabla \bar{\varphi}_{\tau}\right)^{2}=-\eta_{\tau}^{\prime \prime}\left(\bar{\varphi}_{\tau}\right) .
\end{aligned}
$$

Combining these, we obtain

$$
\beta(\nabla \varphi) \partial_{t} \varphi-\operatorname{tr}\left\{D^{2} \alpha(\nabla \varphi) \nabla^{2} \varphi\right\}-\gamma(\nabla \varphi) f \geqslant-\eta_{\tau}^{\prime \prime}\left(\bar{\varphi}_{\tau}\right) \quad \text { at }\left(x_{\tau}, t_{\tau}\right) .
$$

Case 1.1. We verify 3.8 for $\left(x_{0}, t_{0}\right) \in\{(x, t) ; d(x, t)>0\}$. By 3.7 we obtain

$$
-\eta_{\tau}^{\prime \prime}\left(\bar{\varphi}_{\tau}\right) \geqslant-C_{\eta} / \delta \quad \text { at }\left(x_{\tau}, t_{\tau}\right)
$$

We apply this estimate to 3.15 and let $\tau \rightarrow 0$ to find that

$$
\beta(\nabla \varphi) \partial_{t} \varphi-\operatorname{tr}\left\{D^{2} \alpha(\nabla \varphi) \nabla^{2} \varphi\right\}-\gamma(\nabla \varphi) f \geqslant-C_{\eta} / \delta \quad \text { at }\left(x_{0}, t_{0}\right) .
$$

Moreover, by 3.13,

$$
1=\gamma\left(\nabla \bar{\varphi}_{\tau}\right)=\left|\nabla \bar{\varphi}_{\tau}\right| \gamma\left(\frac{\nabla \bar{\varphi}_{\tau}}{\left|\nabla \bar{\varphi}_{\tau}\right|}\right) \geqslant \frac{\left|\nabla \bar{\varphi}_{\tau}\right|}{\Lambda_{\gamma}} \quad \text { at }\left(x_{\tau}, t_{\tau}\right) .
$$


By definition we have $|\nabla \varphi|=\left|\nabla \bar{\varphi}_{\tau}\right| / \rho_{\tau}^{\prime}(\kappa)$ and $1 / \rho_{\tau}^{\prime}(\cdot)=\eta_{\tau}^{\prime}\left(\rho_{\tau}(\cdot)\right) \leqslant C_{\eta}+\tau$. Thus

$$
|\nabla \varphi| \leqslant\left(C_{\eta}+\tau\right)\left|\nabla \bar{\varphi}_{\tau}\right| \leqslant\left(C_{\eta}+\tau\right) \Lambda_{\gamma} \quad \text { at }\left(x_{\tau}, t_{\tau}\right) .
$$

We let $\tau \rightarrow 0$ to conclude that

$$
|\nabla \varphi| \leqslant C_{\eta} \Lambda_{\gamma}=: C_{\gamma} \quad \text { at }\left(x_{0}, t_{0}\right) \text {. }
$$

Case 1.2. We verify (3.9). Since $d$ is lower semicontinuous, there exists a positive constant $\tau_{0}>0$ such that $\tau<\tau_{0}$ implies $d\left(x_{\tau}, t_{\tau}\right)>\delta / 2$. Since $\eta_{\tau}^{\prime \prime}(\sigma)=0$ for $\sigma>\delta / 2$, we obtain

$$
\eta_{\tau}^{\prime \prime}\left(\varphi_{\tau}\left(x_{\tau}, t_{\tau}\right)\right)=\eta_{\tau}^{\prime \prime}\left(d\left(x_{\tau}, t_{\tau}\right)\right)=0 \quad \text { for } \tau<\tau_{0} .
$$

We apply this equality to 3.15 and let $\tau \rightarrow 0$ to deduce that

$$
\beta(\nabla \varphi) \partial_{t} \varphi-\operatorname{tr}\left\{D^{2} \alpha(\nabla \varphi) \nabla^{2} \varphi\right\}-\gamma(\nabla \varphi) f \geqslant 0 \quad \text { at }\left(x_{0}, t_{0}\right) .
$$

Moreover, since $\nabla \varphi=\eta_{\tau}^{\prime}\left(\bar{\varphi}_{\tau}\right) \nabla \bar{\varphi}_{\tau}=(1+\tau) \nabla \bar{\varphi}_{\tau}$, by (3.13) we obtain

$$
1=\gamma\left(\frac{\nabla \varphi}{1+\tau}\right) \quad \text { at }\left(x_{\tau}, t_{\tau}\right)
$$

Letting $\tau \rightarrow 0$ yields $\gamma(\nabla \varphi)=1$ at $\left(x_{0}, t_{0}\right)$.

Case 2. Assume that $d\left(x_{0}, t_{0}\right) \leqslant 0$. Since $d$ is left continuous in time in the sense that

$$
\lim _{x \rightarrow x_{0}} \lim _{t \uparrow t_{0}} d(x, t)=d\left(x_{0}, t_{0}\right),
$$

there exist a positive constant $r_{0}$ and a neighborhood $U_{0}\left(x_{0}\right)$ of $x_{0}$ satisfying

$$
d(x, t) \leqslant \delta / 4 \quad \text { for }(x, t) \in U_{0}\left(x_{0}\right) \times\left(t_{0}-r_{0}, t_{0}\right) .
$$

For $(x, t) \in U_{0}\left(x_{0}\right) \times\left(t_{0}-r_{0}, t_{0}\right)$ we have $\omega(x, t)=-\delta$, i.e., $\omega$ is constant there. This implies

$$
\partial_{t} \varphi\left(x_{0}, t_{0}\right) \geqslant 0, \quad \nabla \varphi\left(x_{0}, t_{0}\right)=0, \quad \nabla^{2} \varphi\left(x_{0}, t_{0}\right) \leqslant 0 .
$$

Since $\nabla \varphi\left(x_{0}, t_{0}\right)=0$, we need to take the upper semicontinuous envelope of the equation 3.8. We observe that

$$
\beta(p) \partial_{t} \varphi \geqslant 0, \quad-\operatorname{tr}\left\{D^{2} \alpha(p) \nabla^{2} \varphi\left(x_{0}, t_{0}\right)\right\} \geqslant 0 \quad \text { for } p \neq 0,
$$

since $\beta>0$ and $\gamma^{2}$ is strictly convex. We have $\lim _{p \rightarrow 0} \gamma(p) f=0$. Therefore

$$
\begin{aligned}
& {\left[\beta(\nabla \varphi) \partial_{t} \varphi-\operatorname{tr}\left\{D^{2} \alpha(\nabla \varphi) \nabla^{2} \varphi\right\}-\gamma(\nabla \varphi) f\right]^{*}\left(x_{0}, t_{0}\right)} \\
& \geqslant \varliminf_{p \rightarrow 0}\left[\beta(p) \partial_{t} \varphi\left(x_{0}, t_{0}\right)-\operatorname{tr}\left\{D^{2} \alpha(p) \nabla^{2} \varphi\left(x_{0}, t_{0}\right)\right\}-\gamma(p) f\right] \geqslant 0 \geqslant-C_{\eta} / \delta .
\end{aligned}
$$

Moreover, $\left|\nabla \varphi\left(x_{0}, t_{0}\right)\right|=0 \leqslant C_{\gamma}$.

We next give an estimate of $\partial_{t} \omega$ by using Lemma 3.3 
LEMmA 3.5 Assume that $\beta, \gamma$ and $f$ satisfy $(\beta 1)-(\beta 3),(\gamma 1)-(\gamma 5)$, and $(f 1)$, respectively. There exists a positive constant $C_{\beta, f}$ which depends only on $n, \Lambda_{\beta}$, and $\Lambda_{f}$ such that the truncated anisotropic distance function $\omega(x, t)=\eta(d(x, t))$ is a viscosity supersolution of

$$
\partial_{t} \omega=-C_{\beta, f} / \delta \quad \text { in } \mathbb{R}^{n} \times(0, T) .
$$

Proof. We continue to use the notations of the proof of Lemma 3.4

Case 1. Assume that $d\left(x_{0}, t_{0}\right)>\delta / 8$. Since $d$ is lower semicontinuous, there exists a positive constant $\tau_{1}>0$ satisfying

$$
d\left(x_{\tau}, t_{\tau}\right) \geqslant \delta / 8 \quad \text { for } \tau<\tau_{1} .
$$

Then Lemma 3.3 shows that

$$
\partial_{t} \bar{\varphi}_{\tau} \geqslant-L_{\beta, f}(1+1 / d) \quad \text { at }\left(x_{\tau}, t_{\tau}\right) .
$$

Since $\partial_{t} \bar{\varphi}_{\tau}=\rho_{\tau}^{\prime}(\kappa) \partial_{t} \varphi$ and $d\left(x_{\tau}, t_{\tau}\right) \geqslant \delta / 8$, we obtain

$$
\partial_{t} \varphi \geqslant-\frac{L_{\beta, f}}{\rho_{\tau}^{\prime}(\kappa)}\left(1+\frac{8}{\delta}\right) \geqslant-\frac{9 L_{\beta, f}\left(\left\|\eta^{\prime}\right\|_{\infty}+1\right)}{\delta} \quad \text { at }\left(x_{\tau}, t_{\tau}\right),
$$

where we have invoked that $\tau \in(0,1)$ and $\delta \in(0,1)$. We take $C_{\beta, f}=9 L_{\beta, f}\left(C_{\eta}+1\right)$ and let $\tau \rightarrow 0$ to reach the desired conclusion in $\{(x, t) ; d(x, t) \geqslant \delta / 8\}$.

Case 2. Assume that $d\left(x_{0}, t_{0}\right) \leqslant \delta / 8$. By a similar argument to Case 2 in the proof of Lemma 3.4 . we obtain, in particular,

$$
\partial_{t} \varphi\left(x_{0}, t_{0}\right) \geqslant 0 \geqslant-C_{\beta, f} / \delta .
$$

\section{Construction of supersolutions for estimating the internal layer}

In this section we construct a supersolution for estimating a solution of (2.9). The basic strategy follows the construction of [ESS].

Let $u$ be a viscosity solution of (2.4) with initial data $u(x, 0)=d_{0}(x)$. The set $\Gamma_{t}^{\delta}=$ $\{x ; u(x, t)=-2 \delta\}$ is also a generalized solution of 2.1 . So we introduce an anisotropic signed distance function $d_{\delta}(x, t)$ defined by

$$
d_{\delta}(x, t)= \begin{cases}\Xi\left(x, \Gamma_{t}^{\delta}\right) & \text { if } x \in\{y ; u(y, t) \geqslant-2 \delta\}, \\ -\Xi\left(x, \Gamma_{t}^{\delta}\right) & \text { if } x \in\{y ; u(y, t)<-2 \delta\} .\end{cases}
$$

By the definition of $d_{\delta}$ the properties in $\S 3$ still hold for $d_{\delta}$ and $\omega_{\delta}=\eta\left(d_{\delta}\right)$.

Combining this and the traveling wave of $\$ 2.3$ we introduce a candidate for our viscosity supersolution for (2.9). We define a function $\psi_{\varepsilon}: \mathbb{R}^{n} \times(0, T) \rightarrow \mathbb{R}$ by

$$
\psi_{\varepsilon}(x, t)=Q\left(\frac{\omega_{\delta}(x, t)+K_{1} t}{\varepsilon}\right)+\varepsilon K_{2},
$$

where $K_{1}$ and $K_{2}$ are positive constants to be determined later. We shall verify the following propositions. 
Proposition 4.1 Assume that $\beta, \gamma, f$ and $\varepsilon$ satisfy $(\beta 1)-(\beta 3),(\gamma 1)-(\gamma 5),(f 1)$ and $(\varepsilon 1)$, respectively. Then, for $\delta>0$, there exist positive constants $K_{1}=K_{1}(\delta), K_{2}=K_{2}\left(\delta, \Lambda_{\beta}, \Lambda_{f}\right)$ and $\varepsilon_{0}=\varepsilon_{0}\left(\delta, \Lambda_{\beta}, \Lambda_{\gamma}, \Lambda_{f}\right)$ such that $\psi_{\varepsilon}$ is a viscosity supersolution of

$$
\beta\left(\nabla \psi_{\varepsilon}\right) \partial_{t} \psi_{\varepsilon}-\operatorname{div}\left\{\gamma\left(\nabla \psi_{\varepsilon}\right) \xi\left(\nabla \psi_{\varepsilon}\right)\right\}+\frac{1}{\varepsilon^{2}}\left(W^{\prime}\left(\psi_{\varepsilon}\right)-\varepsilon \lambda f\right)=\frac{K_{\beta, \delta, f}}{\varepsilon} \quad \text { in } \mathbb{R}^{n} \times(0, T)
$$

provided that $\varepsilon \in\left(0, \varepsilon_{0}\right)$, where $K_{\beta, \delta, f}$ is a numerical positive constant depending only on $\Lambda_{\beta}, \Lambda_{f}$ and $\delta$.

This proposition says not only that $\psi_{\varepsilon}$ is a viscosity supersolution of (2.6) but also that the left hand side of 2.6 with $v=\psi_{\varepsilon}$ increases to $+\infty$ like $1 / \varepsilon$.

Proof. We shall take $\varepsilon_{0}$ small seven times in our proof: in 4.5, 4.10, 4.11, 4.12, 4.14, , 4.18, and (4.19). It suffices to take the minimum of these choices to obtain the conclusion.

Let $\left(x_{\varepsilon}, t_{\varepsilon}\right) \in \mathbb{R}^{n} \times(0, T)$ and let $\varphi \in C^{2}\left(\mathbb{R}^{n} \times(0, T)\right)$ satisfy

$$
\psi_{\varepsilon}(x, t)-\varphi(x, t)>\psi_{\varepsilon}\left(x_{\varepsilon}, t_{\varepsilon}\right)-\varphi\left(x_{\varepsilon}, t_{\varepsilon}\right)=0 \quad \text { whenever }(x, t) \neq\left(x_{\varepsilon}, t_{\varepsilon}\right) .
$$

Since $Q^{\prime}>0$ in $\mathbb{R}$ we have $Q^{-1} \in C^{\infty}(\mathbb{R})$ and $\left(Q^{-1}\right)^{\prime}>0$. Set

$$
\tilde{\varphi}(x, t)=\varepsilon Q^{-1}\left(\varphi(x, t)-\varepsilon K_{2}\right)-K_{1} t
$$

Then $\tilde{\varphi} \in C^{2,1}\left(\mathbb{R}^{n} \times(0, T)\right)$ and

$$
\begin{aligned}
& \omega_{\delta}(x, t)-\tilde{\varphi}(x, t) \geqslant \omega_{\delta}\left(x_{\varepsilon}, t_{\varepsilon}\right)-\tilde{\varphi}\left(x_{\varepsilon}, t_{\varepsilon}\right) \quad \text { for }(x, t) \in \mathbb{R}^{n} \times(0, T), \\
& \varphi(x, t)=Q\left(\frac{\tilde{\varphi}(x, t)+K_{1} t}{\varepsilon}\right)+\varepsilon K_{2} .
\end{aligned}
$$

By straightforward calculation we obtain

$$
\begin{aligned}
\partial_{t} \varphi & =\frac{1}{\varepsilon} Q^{\prime}(h)\left(\partial_{t} \tilde{\varphi}+K_{1}\right), \\
\nabla \varphi & =\frac{1}{\varepsilon} Q^{\prime}(h) \nabla \varphi, \\
\nabla^{2} \varphi & =\frac{1}{\varepsilon^{2}} Q^{\prime \prime}(h) \nabla \tilde{\varphi} \otimes \nabla \tilde{\varphi}+\frac{1}{\varepsilon} Q^{\prime}(h) \nabla^{2} \tilde{\varphi},
\end{aligned}
$$

where $h=h(x, t)=\left(\tilde{\varphi}(x, t)+K_{1} t\right) / \varepsilon$. Moreover,

$$
W^{\prime}\left(\psi_{\varepsilon}\right)=W^{\prime}(\varphi)=W^{\prime}(Q(h))+\varepsilon K_{2} W^{\prime \prime}(Q(h))+O\left(\varepsilon^{2} K_{2}^{2}\right) \quad \text { at }\left(x_{\varepsilon}, t_{\varepsilon}\right)
$$

as $\varepsilon \rightarrow 0$. We now take $\varepsilon_{0}=\varepsilon_{0}\left(K_{2}\right)$ small so that

$$
\left|\varepsilon K_{2}\right| \leqslant 1 \quad \text { provided } \varepsilon \in\left(0, \varepsilon_{0}\right) .
$$

Case 1. We assume that $\left(x_{\varepsilon}, t_{\varepsilon}\right)$ satisfies $d_{\delta}\left(x_{\varepsilon}, t_{\varepsilon}\right)>\delta / 2$. By Lemma 3.4 we have

$$
\left.\begin{array}{l}
\gamma(\nabla \tilde{\varphi})=1, \text { in particular } \nabla \tilde{\varphi} \neq 0, \\
\beta(\nabla \tilde{\varphi}) \partial_{t} \tilde{\varphi}-\operatorname{tr}\left\{D^{2} \alpha(\nabla \tilde{\varphi}) \nabla^{2} \tilde{\varphi}\right\}-\gamma(\nabla \tilde{\varphi}) f \geqslant 0
\end{array}\right\} \quad \text { at }\left(x_{\varepsilon}, t_{\varepsilon}\right)
$$


We observe that $\nabla \varphi \neq 0$ since $\nabla \tilde{\varphi} \neq 0$. We set

$$
R_{\varepsilon}=R_{\varepsilon}(x, t)=\beta(\nabla \varphi) \partial_{t} \varphi-\operatorname{tr}\left\{D^{2} \alpha(\nabla \varphi) \nabla^{2} \varphi\right\}+\frac{1}{\varepsilon^{2}}\left(W^{\prime}\left(\psi_{\varepsilon}\right)-\varepsilon \lambda f\right) .
$$

Our aim is to show that there exists a positive constant $K_{\beta, \delta, f}$, which depends only on $\Lambda_{\beta}, \Lambda_{f}$ and $\delta$, satisfying $R_{\varepsilon} \geqslant K_{\beta, \delta, f} / \varepsilon$ at $\left(x_{\varepsilon}, t_{\varepsilon}\right)$.

By the homogeneity of $\beta, \gamma$ and $\alpha$,

$$
\beta(\nabla \varphi)=\beta(\nabla \tilde{\varphi}), \quad \gamma(\nabla \varphi)=\frac{1}{\varepsilon} Q^{\prime}(h) \gamma(\nabla \tilde{\varphi}), \quad D^{2} \alpha(\nabla \varphi)=D^{2} \alpha(\nabla \tilde{\varphi}) .
$$

By 4.1 - 4.3 we now obtain

$$
\beta(\nabla \varphi) \partial_{t} \varphi=\beta(\nabla \tilde{\varphi}) Q^{\prime}(h) \frac{\partial_{t} \tilde{\varphi}+K_{1}}{\varepsilon},
$$

and

$$
\begin{aligned}
\operatorname{tr}\left\{D^{2} \alpha(\nabla \varphi) \nabla^{2} \varphi\right\} & =\frac{Q^{\prime \prime}(h)}{\varepsilon^{2}}\left\langle D^{2} \alpha(\nabla \tilde{\varphi}) \nabla \tilde{\varphi}, \nabla \tilde{\varphi}\right\rangle+\frac{Q^{\prime}(h)}{\varepsilon} \operatorname{tr}\left\{D^{2} \alpha(\nabla \tilde{\varphi}) \nabla^{2} \tilde{\varphi}\right\} \\
& =\frac{Q^{\prime \prime}(h)}{\varepsilon^{2}} \gamma(\nabla \tilde{\varphi})^{2}+\frac{Q^{\prime}(h)}{\varepsilon} \operatorname{tr}\left\{D^{2} \alpha(\nabla \tilde{\varphi}) \nabla^{2} \tilde{\varphi}\right\} .
\end{aligned}
$$

Here we have invoked the property that $\left\langle D^{2} \alpha(p) p, p\right\rangle=2 \alpha(p)=\gamma(p)^{2}$ for $p \neq 0$ since $\alpha$ is positively homogeneous of degree 2 . Combining (4.4) and the above, we conclude that

$$
\begin{aligned}
R_{\varepsilon} & =\frac{1}{\varepsilon^{2}} I_{-2}+\frac{1}{\varepsilon} I_{-1}+O\left(K_{2}^{2}\right), \\
I_{-2} & =-Q^{\prime \prime}(h) \gamma(\nabla \tilde{\varphi})^{2}+W^{\prime}(Q(h))-\varepsilon \lambda f, \\
I_{-1} & =K_{2} W^{\prime \prime}(Q(h))+Q^{\prime}(h)\left[\beta(\nabla \tilde{\varphi}) K_{1}+\beta(\nabla \tilde{\varphi}) \partial_{t} \tilde{\varphi}-\operatorname{tr}\left\{D^{2} \alpha(\nabla \tilde{\varphi}) \nabla^{2} \tilde{\varphi}\right\}\right] .
\end{aligned}
$$

By $[2.10]$ and since $\gamma(\nabla \tilde{\varphi})=1$,

$$
I_{-2}=-Q^{\prime \prime}(h)+W^{\prime}(Q(h))-\varepsilon \lambda f=c Q^{\prime}(h) .
$$

Then, by using (4.6), we obtain

$$
\begin{aligned}
R_{\varepsilon}= & \frac{1}{\varepsilon}\left(K_{2} W^{\prime \prime}(Q(h))+Q^{\prime}(h)\left[f+\frac{c}{\varepsilon}+\beta(\nabla \tilde{\varphi}) K_{1}\right.\right. \\
& \left.\left.+\beta(\nabla \tilde{\varphi}) \partial_{t} \tilde{\varphi}-\operatorname{tr}\left\{D^{2} \alpha(\nabla \tilde{\varphi}) \nabla^{2} \tilde{\varphi}\right\}-\gamma(\nabla \tilde{\varphi}) f\right]\right)+O\left(K_{2}^{2}\right) \\
\geqslant & \frac{1}{\varepsilon}\left[K_{2} W^{\prime \prime}(Q(h))+Q^{\prime}(h)\left(f+\frac{c}{\varepsilon}+\frac{K_{1}}{\Lambda_{\beta}}\right)\right]+O\left(K_{2}^{2}\right) \quad \text { at }\left(x_{\varepsilon}, t_{\varepsilon}\right) .
\end{aligned}
$$

We now determine $K_{1}$. We take

$$
K_{1}=\frac{\delta}{4 T} .
$$

The reason for this choice is clarified in Case 2. By Proposition 2.1 we take $\varepsilon_{0}=\varepsilon_{0}\left(\delta, \Lambda_{f}\right)$ smaller so that

$$
f+\frac{c}{\varepsilon} \geqslant-\frac{K_{1}}{2 \Lambda_{\beta}}=-\frac{\delta}{8 \Lambda_{\beta} T} \quad \text { provided } \varepsilon \in\left(0, \varepsilon_{0}\right)
$$


Then we obtain

$$
R_{\varepsilon} \geqslant \frac{1}{\varepsilon}\left[K_{2} W^{\prime \prime}(Q(h))+Q^{\prime}(h) C_{\beta, \delta}\right]+O\left(K_{2}^{2}\right) \quad \text { at }\left(x_{\varepsilon}, t_{\varepsilon}\right),
$$

where $C_{\beta, \delta}=\delta /\left(8 \Lambda_{\beta} T\right)$.

Now we determine $K_{2}$. It will enable us to estimate $R_{\varepsilon}$ in the case that $W^{\prime \prime}(Q(h))<0$. The basic strategy comes from the fact that $Q(\sigma) \rightarrow \tanh \sigma$ uniformly with respect to $\sigma \in \mathbb{R}$ and $f$ satisfying $(f 1)$ as $\varepsilon \rightarrow 0, W^{\prime}(\tanh \sigma) \geqslant 0$ for large enough $|\sigma|$, and we have a local uniform bound of $Q^{\prime}$ from below with respect to $f$ and $\varepsilon$ satisfying $(f 1)$ and $(\varepsilon 1)$.

By Proposition 2.1 ii) we take $\varepsilon_{0}=\varepsilon_{0}\left(\Lambda_{f}\right)$ smaller so that

$$
|Q(\sigma)-\tanh \sigma| \leqslant \frac{1}{2}\left(1-\frac{1}{\sqrt{2}}\right)=: v \quad \text { for } \sigma \in \mathbb{R} \text { provided } \varepsilon \in\left(0, \varepsilon_{0}\right) .
$$

We take $b=-\sup \{\sigma ; \tanh \sigma+v \leqslant-1 / \sqrt{2}\}=\inf \{\sigma ; \tanh \sigma-v \geqslant 1 / \sqrt{2}\}$ and let

$$
\begin{aligned}
K_{2} & =\frac{a_{2} C_{\beta, \delta}}{2 a_{1}}, \\
a_{1} & =\left|\inf _{|\sigma| \leqslant 1+\nu} W^{\prime \prime}(\sigma)\right|, \quad a_{2}=\inf \left\{Q^{\prime}(\sigma) ;|\sigma| \leqslant b, \varepsilon \in(0, \bar{\varepsilon}),|f| \leqslant \Lambda_{f}\right\} .
\end{aligned}
$$

We remark that $a_{2}>0$ exists by Proposition 2.1 iii). Moreover, $\varepsilon_{0}=\varepsilon_{0}\left(K_{2}\right)$ implies that $\varepsilon_{0}$ depends on $\delta, \Lambda_{\beta}$ and $\Lambda_{f}$.

We consider two subcases.

Case 1.1. Assume that $\left(x_{\varepsilon}, t_{\varepsilon}\right) \in\{(x, t) ;|h(x, t)| \leqslant b\}$. Then $Q\left(h\left(x_{\varepsilon}, t_{\varepsilon}\right)\right) \leqslant 1$. Therefore

$$
R_{\varepsilon} \geqslant \frac{1}{\varepsilon}\left(-K_{2} a_{1}+a_{2} C_{\beta, \delta}\right)+O\left(K_{2}^{2}\right) \geqslant \frac{a_{2} C_{\beta, \delta}}{2 \varepsilon}+O\left(K_{2}^{2}\right) \quad \text { at }\left(x_{\varepsilon}, t_{\varepsilon}\right) .
$$

This is why we take $K_{2}$ as above. We now take $\varepsilon_{0}=\varepsilon_{0}\left(\delta, \Lambda_{\beta}, \Lambda_{f}\right)$ smaller so that

$$
\left|\varepsilon O\left(K_{2}^{2}\right)\right| \leqslant a_{2} C_{\beta, \delta} / 4 \quad \text { provided } \varepsilon \in\left(0, \varepsilon_{0}\right) .
$$

Then we obtain

$$
R_{\varepsilon} \geqslant \frac{a_{2} C_{\beta, \delta}}{4 \varepsilon}>0 \quad \text { at }\left(x_{\varepsilon}, t_{\varepsilon}\right)
$$

Case 1.2. Assume that $\left(x_{\varepsilon}, t_{\varepsilon}\right) \in\{(x, t) ;|h(x, t)|>b\}$. Then $W^{\prime \prime}\left(Q\left(h\left(x_{\varepsilon}, t_{\varepsilon}\right)\right)\right)>1$ and

$$
R_{\varepsilon} \geqslant K_{2} / \varepsilon+O\left(K_{2}^{2}\right) \quad \text { at }\left(x_{\varepsilon}, t_{\varepsilon}\right) .
$$

We now take $\varepsilon_{0}=\varepsilon_{0}\left(\delta, \Lambda_{\beta}, \Lambda_{f}\right)$ smaller so that

$$
\left|\varepsilon O\left(K_{2}^{2}\right)\right| \leqslant K_{2} / 2 \quad \text { provided } \varepsilon \in\left(0, \varepsilon_{0}\right) .
$$

Then we obtain

$$
R_{\varepsilon} \geqslant \frac{K_{2}}{2 \varepsilon}>0 \quad \text { at }\left(x_{\varepsilon}, t_{\varepsilon}\right) .
$$


Case 2. We assume that $\left(x_{\varepsilon}, t_{\varepsilon}\right) \in\left\{(x, t) ; d_{\delta}(x, t) \leqslant \delta / 2\right\}$. By Lemma 3.4 we have

$$
\begin{aligned}
& \left|\nabla \tilde{\varphi}\left(x_{\varepsilon}, t_{\varepsilon}\right)\right| \leqslant C_{\gamma}, \\
& {\left[\beta(\nabla \tilde{\varphi}) \partial_{t} \tilde{\varphi}-\operatorname{tr}\left\{D^{2} \alpha(\nabla \tilde{\varphi}) \nabla^{2} \tilde{\varphi}\right\}-\gamma(\nabla \tilde{\varphi}) f\right]^{*}\left(x_{\varepsilon}, t_{\varepsilon}\right) \geqslant-C_{\eta} / \delta .}
\end{aligned}
$$

We first observe that

$$
h\left(x_{\varepsilon}, t_{\varepsilon}\right)=\frac{\eta\left(d_{\delta}\left(x_{\varepsilon}, t_{\varepsilon}\right)\right)+K_{1} t_{\varepsilon}}{\varepsilon} \leqslant-\frac{\delta}{4 \varepsilon}<0,
$$

i.e., $h \rightarrow-\infty$ as $\varepsilon \rightarrow 0$. This is why we take $K_{1}$ as in 4.10 . Therefore we take $\varepsilon_{0}=\varepsilon_{0}(\delta)$ smaller so that

$$
\left(x_{\varepsilon}, t_{\varepsilon}\right) \in\left\{(x, t) ; W^{\prime \prime}(Q(h(x, t))) \geqslant 1\right\} \quad \text { for }\left(x_{\varepsilon}, t_{\varepsilon}\right) \in\left\{(x, t) ; d_{\delta}(x, t)<\delta / 2\right\}
$$

provided $\varepsilon \in\left(0, \varepsilon_{0}\right)$.

Case 2.1. Assume that $\nabla \tilde{\varphi}\left(x_{\varepsilon}, t_{\varepsilon}\right) \neq 0$. By the same argument as in Case 1.1, it suffices to see

$$
R_{\varepsilon}=\frac{1}{\varepsilon^{2}} I_{-2}+\frac{1}{\varepsilon} I_{-1}+O\left(K_{2}^{2}\right) \geqslant K_{\delta, \beta, f} / \delta \quad \text { at }\left(x_{\varepsilon}, t_{\varepsilon}\right),
$$

where $R_{\varepsilon}, I_{-2}$ and $I_{-1}$ are defined by (4.7), (4.8), and 4.9), respectively. We remark that $\gamma\left(\nabla \tilde{\varphi}\left(x_{\varepsilon}, t_{\varepsilon}\right)\right) \neq 1$ in this case. Therefore

$$
I_{-2}=-Q^{\prime \prime}(h)\left(\gamma(\nabla \tilde{\varphi})^{2}-1\right)+c Q^{\prime}(h) .
$$

Then we observe from 4.16

$$
\begin{aligned}
R_{\varepsilon} \geqslant & -\frac{1}{\varepsilon^{2}} Q^{\prime \prime}(h)\left(\gamma(\nabla \tilde{\varphi})^{2}-1\right)+\frac{1}{\varepsilon}\left[K_{2}+Q^{\prime}(h)\left\{C_{\beta, \delta}+(\gamma(\nabla \tilde{\varphi})-1) f-\frac{C_{\eta}}{\delta}\right\}\right] \\
& +O\left(K_{2}^{2}\right) \quad \text { at }\left(x_{\varepsilon}, t_{\varepsilon}\right) .
\end{aligned}
$$

By the homogeneity of $\gamma$ we obtain

$$
\gamma(\nabla \tilde{\varphi})=|\nabla \tilde{\varphi}| \gamma\left(\frac{\nabla \tilde{\varphi}}{|\nabla \tilde{\varphi}|}\right) \leqslant C_{\gamma} \Lambda_{\gamma} \quad \text { at }\left(x_{\varepsilon}, t_{\varepsilon}\right) .
$$

Therefore

$$
R_{\varepsilon} \geqslant-\frac{1}{\varepsilon^{2}}\left(C_{\gamma}^{2} \Lambda_{\gamma}^{2}+1\right)\left|Q^{\prime \prime}(h)\right|+\frac{1}{\varepsilon}\left\{K_{2}-C_{\beta, \gamma, \delta}\left|Q^{\prime}(h)\right|\right\}+O\left(K_{2}^{2}\right) \quad \text { at }\left(x_{\varepsilon}, t_{\varepsilon}\right),
$$

where $C_{\beta, \gamma, \delta}:=C_{\beta, \delta}+\left(C_{\gamma} \Lambda_{\gamma}+1\right)|f|+C_{\eta} / \delta$ is a constant. By (2.12) and 4.17),

$$
\begin{aligned}
R_{\varepsilon} & \geqslant \frac{1}{\varepsilon}\left\{K_{2}-\left(C_{\beta, \gamma, \delta}+\frac{C_{\gamma}^{2} \Lambda_{\gamma}^{2}+1}{\varepsilon}\right) C_{1} \exp \left(-C_{2}|h|\right)\right\}+O\left(K_{2}^{2}\right) \\
& \geqslant \frac{1}{\varepsilon}\left\{K_{2}-\left(C_{\beta, \gamma, \delta}+\frac{C_{\gamma}^{2} \Lambda_{\gamma}^{2}+1}{\varepsilon}\right) C_{1} \exp \left(-\frac{C_{2} \delta}{4 \varepsilon}\right)\right\}+O\left(K_{2}^{2}\right) \quad \text { at }\left(x_{\varepsilon}, t_{\varepsilon}\right) .
\end{aligned}
$$


We take $\varepsilon_{0}=\varepsilon_{0}\left(\delta, \Lambda_{\beta}, \Lambda_{\gamma}, \Lambda_{f}\right)$ smaller so that

$$
\left.\begin{array}{l}
\left|\left(C_{\beta, \gamma, \delta}+\frac{C_{\gamma}^{2} \Lambda_{\gamma}^{2}+1}{\varepsilon}\right) C_{1} \exp \left(-\frac{C_{2} \delta}{4 \varepsilon}\right)\right| \leqslant \frac{K_{2}}{4} \\
\left|\varepsilon O\left(K_{2}^{2}\right)\right| \leqslant \frac{K_{2}}{4}
\end{array}\right\} \quad \text { provided } \varepsilon \in\left(0, \varepsilon_{0}\right) .
$$

Then we obtain

$$
R_{\varepsilon} \geqslant \frac{K_{2}}{2 \varepsilon}>0 \quad \text { at }\left(x_{\varepsilon}, t_{\varepsilon}\right) .
$$

Case 2.2. Assume that $\nabla \tilde{\varphi}\left(x_{\varepsilon}, t_{\varepsilon}\right)=0$. We need to consider the equations in weak sense. We now set $\hat{\sigma}_{\varepsilon}=\psi_{\varepsilon}\left(x_{\varepsilon}, t_{\varepsilon}\right), \hat{s}_{\varepsilon}=\partial_{t} \varphi\left(x_{\varepsilon}, t_{\varepsilon}\right), \hat{p}_{\varepsilon}=\nabla \varphi\left(x_{\varepsilon}, t_{\varepsilon}\right), \hat{X}_{\varepsilon}=\nabla^{2} \varphi\left(x_{\varepsilon}, t_{\varepsilon}\right)$, and

$$
\begin{aligned}
\bar{R}_{\varepsilon}=\varlimsup_{r \rightarrow 0}\left\{\beta(p) s-\operatorname{tr}\left\{D^{2} \alpha(p) X\right\}\right. & +\frac{1}{\varepsilon^{2}}\left(W^{\prime}(\sigma)-\varepsilon \lambda f\right) ; \\
& \left.\left|\sigma-\hat{\sigma}_{\varepsilon}\right|<r,\left|s-\hat{s}_{\varepsilon}\right|<r,\left|p-\hat{p}_{\varepsilon}\right|<r,\left|X-\hat{X}_{\varepsilon}\right|<r\right\},
\end{aligned}
$$

We shall prove $\bar{R}_{\varepsilon} \geqslant K_{\beta, \delta, f} / \delta$. By 4.16 there exists a sequence $\left\{\left(\tau_{j}, q_{j}, Y_{j}\right)\right\}_{j=1}^{\infty}$ satisfying

$$
\begin{aligned}
& \lim _{j \rightarrow \infty}\left(\tau_{j}, q_{j}, Y_{j}\right)=\left(\partial_{t} \tilde{\varphi}\left(x_{\varepsilon}, t_{\varepsilon}\right), 0, \nabla^{2} \tilde{\varphi}\left(x_{\varepsilon}, t_{\varepsilon}\right)\right), \\
& q_{j} \neq 0, \quad \varlimsup_{j \rightarrow \infty}\left|q_{j}\right| \leqslant C_{\gamma}, \\
& \lim _{j \rightarrow \infty}\left[\beta\left(q_{j}\right) \tau_{j}-\operatorname{tr}\left\{D^{2} \alpha\left(q_{j}\right) Y_{j}\right\}-\gamma\left(q_{j}\right) f\right] \geqslant 0 .
\end{aligned}
$$

We now set

$$
\left\{\begin{aligned}
\sigma_{j} & =Q\left(h\left(x_{\varepsilon}, t_{\varepsilon}\right)\right)+\varepsilon K_{2}=\hat{\sigma}_{\varepsilon}, \\
s_{j} & =\frac{1}{\varepsilon} Q^{\prime}\left(h\left(x_{\varepsilon}, t_{\varepsilon}\right)\right)\left(\tau_{j}+K_{1}\right) \rightarrow \hat{s}_{\varepsilon}, \\
p_{j} & =\frac{1}{\varepsilon} Q^{\prime}\left(h\left(x_{\varepsilon}, t_{\varepsilon}\right) q_{j} \rightarrow 0=\hat{p}_{\varepsilon},\right. \\
X_{j} & =\frac{1}{\varepsilon^{2}} Q^{\prime \prime}\left(h\left(x_{\varepsilon}, t_{\varepsilon}\right)\right) q_{j} \otimes q_{j}+\frac{1}{\varepsilon} Q^{\prime}\left(h\left(x_{\varepsilon}, t_{\varepsilon}\right)\right) Y_{j} \rightarrow \hat{X}_{\varepsilon},
\end{aligned}\right.
$$

where the limits are taken as $j \rightarrow \infty$. Moreover, let

$$
R_{\varepsilon}^{j}:=\beta\left(p_{j}\right) s_{j}-\operatorname{tr}\left\{D^{2} \alpha\left(p_{j}\right) X_{j}\right\}+\frac{1}{\varepsilon^{2}}\left(W^{\prime}\left(\sigma_{j}\right)-\varepsilon \lambda f\right) .
$$

From the arguments in Case 2.1 it follows that

$$
\bar{R}_{\varepsilon} \geqslant \lim _{j \rightarrow \infty} R_{\varepsilon}^{j} \geqslant \frac{K_{2}}{2 \varepsilon}>0
$$

Set $K_{\beta, \delta, f}=\min \left\{K_{2} / 2, a_{2} C_{\beta, \delta} / 4\right\}$. Then we conclude from (4.13), 4.15), 4.20), and 4.22\} that

$$
\left[\beta(\nabla \varphi) \partial_{t} \varphi-\operatorname{tr}\left\{D^{2} \alpha(\nabla \varphi) \nabla^{2} \varphi\right\}+\frac{1}{\varepsilon^{2}}\left(W^{\prime}\left(\psi_{\varepsilon}\right)-\varepsilon \lambda f\right)\right]^{*}\left(x_{\varepsilon}, t_{\varepsilon}\right) \geqslant \frac{K_{\beta, \delta, f}}{\varepsilon}>0 .
$$

We are now in a position to show that $\psi_{\varepsilon}$ is a viscosity supersolution of [2.9]. 
Proposition 4.2 Assume that $\beta, \gamma, f$ and $\varepsilon$ satisfy $(\beta 1)-(\beta 3),(\gamma 1)-(\gamma 5),(f 1)$ and $(\varepsilon 1)$, respectively. Then, for $\delta>0$, there exists a positive constant $\varepsilon_{1}=\varepsilon_{1}\left(\delta, \Lambda_{\beta}, \Lambda_{\gamma}, \Lambda_{f}\right)$ such that $\psi_{\varepsilon}$ is a viscosity supersolution of $[2.9]$ in $\mathbb{R}^{n} \times(0, T)$ provided that $\varepsilon \in\left(0, \varepsilon_{1}\right)$.

Proof. We continue the proof from Proposition 4.1. We first fix $\varepsilon_{1}<\varepsilon_{0}$. In this proof, we take $\varepsilon_{1}$ twice, in 4.25) and (4.26). It suffices to take their minimum.

Case 1. We assume that $\left(x_{\varepsilon}, t_{\varepsilon}\right) \in\left\{(x, t) ; d_{\delta}(x, t)>\delta / 2\right\}$. Since $\gamma(\nabla \tilde{\varphi})=1$ we have $\nabla \varphi \neq 0$. We now set

$$
\tilde{R}_{\varepsilon}=\tilde{\beta}(\nabla \varphi) \partial_{t} \varphi-\operatorname{tr}\left\{D^{2} \alpha(\nabla \varphi) \nabla^{2} \varphi\right\}+\frac{1}{\varepsilon^{2}}\left(W^{\prime}(\varphi)-\varepsilon \lambda f\right)
$$

Our aim is to show $\tilde{R}_{\varepsilon} \geqslant 0$ at $\left(x_{\varepsilon}, t_{\varepsilon}\right)$. By straightforward calculation we obtain

$$
\begin{aligned}
\tilde{R}_{\varepsilon} & =R_{\varepsilon}+\tilde{R}_{\varepsilon}-R_{\varepsilon} \geqslant \frac{K_{\beta, \delta, f}}{\varepsilon}+(\tilde{\beta}(\nabla \varphi)-\beta(\nabla \varphi)) \partial_{t} \varphi \\
& =\frac{1}{\varepsilon}\left[K_{\beta, \delta, f}+Q^{\prime}(h) \zeta(|\nabla \varphi|)\left(\Lambda_{\beta}-\beta(\nabla \varphi)\right)\left(\partial_{t} \tilde{\varphi}+K_{1}\right)\right] \quad \text { at }\left(x_{\varepsilon}, t_{\varepsilon}\right) .
\end{aligned}
$$

We observe that

$$
Q^{\prime}(h) \zeta(|\nabla \varphi|)\left(\Lambda_{\beta}-\beta(\nabla \varphi)\right) K_{1} \geqslant 0 .
$$

Moreover, from Lemma 3.5 we obtain

$$
\begin{aligned}
Q^{\prime}(h) \zeta(|\nabla \varphi|)\left(\Lambda_{\beta}-\beta(\nabla \varphi)\right) \partial_{t} \tilde{\varphi} & \geqslant-Q^{\prime}(h) \zeta(|\nabla \varphi|)\left(\Lambda_{\beta}-\beta(\nabla \varphi)\right) \frac{C_{\beta}}{\delta} \\
& \geqslant-Q^{\prime}(h) \zeta(|\nabla \varphi|)\left(\Lambda_{\beta}-\frac{1}{\Lambda_{\beta}}\right) \frac{C_{\beta}}{\delta} \quad \text { at }\left(x_{\varepsilon}, t_{\varepsilon}\right)
\end{aligned}
$$

Thus

$$
\tilde{R}_{\varepsilon} \geqslant \frac{1}{\varepsilon}\left[K_{\beta, \delta, f}-M_{\beta, \delta} Q^{\prime}(h) \zeta(|\nabla \varphi|)\right] \quad \text { at }\left(x_{\varepsilon}, t_{\varepsilon}\right),
$$

where $M_{\beta, \delta}:=\left(\Lambda_{\beta}-1 / \Lambda_{\beta}\right) C_{\beta} / \delta$. We now study $\tilde{R}_{\varepsilon}$ in two cases.

Case 1.1. Assume that $\left(x_{\varepsilon}, t_{\varepsilon}\right) \in\left\{(x, t) ; M_{\beta, \delta} Q^{\prime}(h(x, t))<K_{\beta, \delta, f} / 2\right\}$. In this case it is easy to see that

$$
\tilde{R}_{\varepsilon} \geqslant \frac{K_{\beta, \delta, f}}{2 \varepsilon}>0 \quad \text { at }\left(x_{\varepsilon}, t_{\varepsilon}\right) .
$$

Case 1.2. Assume that $\left(x_{\varepsilon}, t_{\varepsilon}\right) \in\left\{(x, t) ; M_{\beta, \delta} Q^{\prime}(h(x, t)) \geqslant K_{\beta, \delta, f} / 2\right\}$. We remark that $|\nabla \varphi|=$ $Q^{\prime}(h)|\nabla \tilde{\varphi}| / \varepsilon$. Since $\gamma(\nabla \tilde{\varphi})=1$ we obtain

$$
|\nabla \tilde{\varphi}| \geqslant 1 / \Lambda_{\gamma} \quad \text { at }\left(x_{\varepsilon}, t_{\varepsilon}\right) .
$$

Hence

$$
|\nabla \varphi|=\frac{Q^{\prime}(h)|\nabla \tilde{\varphi}|}{\varepsilon} \geqslant \frac{K_{\beta, \delta, f}}{2 \varepsilon \Lambda_{\gamma} M_{\beta, \delta}} \quad \text { at }\left(x_{\varepsilon}, t_{\varepsilon}\right) .
$$

We take $\varepsilon_{1}=\varepsilon_{1}\left(\delta, \Lambda_{\beta}, \Lambda_{f}\right)$ smaller so that

$$
\frac{K_{\beta, \delta, f}}{2 \varepsilon \Lambda_{\beta} M_{\beta, \delta}} \geqslant 1 \quad \text { provided } \varepsilon \in\left(0, \varepsilon_{1}\right) .
$$


Then we obtain $|\nabla \varphi| \geqslant 1 \geqslant 3 / 4$, i.e., $\zeta(|\nabla \varphi|)=0$ at $\left(x_{\varepsilon}, t_{\varepsilon}\right)$. Thus

$$
\tilde{R}_{\varepsilon} \geqslant \frac{K_{\beta, \delta, f}}{\varepsilon}>0 \quad \text { at }\left(x_{\varepsilon}, t_{\varepsilon}\right) .
$$

Case 2. We assume that $\left(x_{\varepsilon}, t_{\varepsilon}\right) \in\left\{(x, t) ; d_{\delta}(x, t) \leqslant \delta / 2\right\}$. By (4.17) there exists $\varepsilon_{1}=$ $\varepsilon_{1}\left(\delta, \Lambda_{\beta}, \Lambda_{f}\right)$ satisfying

$$
\left(x_{\varepsilon}, t_{\varepsilon}\right) \in\left\{(x, t) ; M_{\beta, \delta} Q^{\prime}(h(x, t))<K_{\beta, \delta, f} / 2\right\} \quad \text { provided } \varepsilon \in\left(0, \varepsilon_{1}\right) .
$$

We take $\varepsilon_{1}$ satisfying 4.26.

Case 2.1. Assume that $\nabla \varphi\left(x_{\varepsilon}, t_{\varepsilon}\right) \neq 0$. From the same argument as in Case 1.1 of this proof we obtain

$$
\tilde{R}_{\varepsilon} \geqslant \frac{K_{\beta, \delta, f}}{2 \varepsilon}>0 \quad \text { at }\left(x_{\varepsilon}, t_{\varepsilon}\right) .
$$

Case 2.2. Assume that $\nabla \varphi\left(x_{\varepsilon}, t_{\varepsilon}\right)=0$. Since $\overline{\lim }_{j \rightarrow \infty} R_{\varepsilon}^{j} \geqslant K_{\beta, \delta, f} / \varepsilon$, there exists a positive number $N_{1} \in \mathbf{N}$ satisfying

$$
R_{\varepsilon}^{j} \geqslant \frac{7 K_{\beta, \delta, f}}{8 \varepsilon} \quad \text { for } j>N_{1}
$$

by taking a subsequence of $\left\{R_{\varepsilon}^{j}\right\}$ if necessary. Set

$$
\tilde{R}_{\varepsilon}^{j}=\tilde{\beta}\left(p_{j}\right) s_{j}-\operatorname{tr}\left\{D^{2} \alpha\left(p_{j}\right) X_{j}\right\}+\frac{1}{\varepsilon^{2}}\left(W^{\prime}\left(\sigma_{j}\right)-\varepsilon \lambda f\right),
$$

where $\sigma_{j}, s_{j}, p_{j}$ and $X_{j}$ are as in 4.21 . By (4.21) there exists a positive number $N_{2} \in \mathbb{N}$ satisfying

$$
Q^{\prime}\left(h\left(x_{\varepsilon}, t_{\varepsilon}\right)\right) \zeta\left(\left|p_{j}\right|\right)\left(\Lambda_{\beta}-\frac{1}{\Lambda_{\beta}}\right) \tau_{j} \geqslant-\frac{5 K_{\beta, \delta, f}}{8} \text { for } j>N_{2}
$$

since $\tau_{j} \rightarrow \partial_{t} \tilde{\varphi}\left(x_{\varepsilon}, t_{\varepsilon}\right)$ as $j \rightarrow \infty$ and by 4.26 . Then

$$
\tilde{R}_{\varepsilon}^{j}=R_{\varepsilon}^{j}+\tilde{R}_{\varepsilon}^{j}-R_{\varepsilon}^{j} \geqslant \frac{1}{\varepsilon}\left(\frac{7 K_{\beta, \delta, f}}{8}+Q^{\prime}\left(h\left(x_{\varepsilon}, t_{\varepsilon}\right)\right) \zeta\left(\left|p_{j}\right|\right)\left(\Lambda_{\beta}-\frac{1}{\Lambda_{\beta}}\right) \tau_{j}\right) \geqslant \frac{K_{\beta, \delta, f}}{4 \varepsilon}>0
$$

for $j>N=\max \left\{N_{1}, N_{2}\right\}$. We thus conclude that

$$
\left(\tilde{R}_{\varepsilon}\right)^{*}\left(x_{\varepsilon}, t_{\varepsilon}\right) \geqslant \varlimsup_{j \rightarrow \infty} R_{\varepsilon}^{j} \geqslant \frac{K_{\beta, \delta, f}}{4 \varepsilon}>0 .
$$

\section{Uniform estimate}

In this section, we shall prove Theorem 2.2

Proof of Theorem 2.2 Let $v$ be a solution of 2.9) with $v(x, 0)=Q\left(d_{0}(x) / \varepsilon\right)$, and $\psi_{\varepsilon}$ be as defined in $\S 4$. We first verify that, for $\delta>0$,

$$
\psi_{\varepsilon}(x, 0) \geqslant v(x, 0) \quad \text { for } x \in \mathbb{R}^{n} .
$$


We remark that $\omega_{\delta}(x, 0)=\eta\left(d_{\delta}(x, 0)\right) \geqslant d_{0}(x)$. Let $y \in \Gamma_{0}^{\delta}$ be such that $d_{\delta}(x, 0)=\gamma^{\circ}(x-y)$. Then

$$
d_{0}(x)-d_{0}(y) \leqslant \gamma^{\circ}(x-y)=d_{\delta}(x, 0) .
$$

By the definition of $\Gamma_{0}^{\delta}$, we have $d_{0}(y)=-2 \delta$. Thus

$$
d_{\delta}(x, 0) \geqslant d_{0}(x)+2 \delta .
$$

If $d_{\delta}(x, 0) \geqslant \delta / 2$, then

$$
\eta\left(d_{\delta}(x, 0)\right)=d_{\delta}(x, 0)-\delta \geqslant d_{0}(x)+\delta>d_{0}(x)
$$

If $d_{\delta}(x, 0)<\delta / 2$, then

$$
d_{0}(x) \leqslant d_{\delta}(x, 0)-2 \delta<-3 \delta / 2<-\delta \leqslant \eta\left(d_{\delta}(x, 0)\right) .
$$

Thus

$$
\psi_{\varepsilon}(x, 0)=Q\left(\frac{\omega_{\delta}(x, 0)}{\varepsilon}\right)+\varepsilon K_{2} \geqslant Q\left(\frac{d_{0}(x)}{\varepsilon}\right)=v(x, 0) .
$$

By the comparison principle and (5.1) we obtain, for $\delta>0$,

$$
\psi_{\varepsilon}(x, t) \geqslant v(x, t) \quad \text { for }(x, t) \in \mathbb{R}^{n} \times(0, T) .
$$

Fix $\theta>0$. We take $\delta$ satisfying $d_{\delta}(x, t)<0$ if $d(x, t)<-\theta$. We recall (4.17), that is,

$$
\frac{\omega_{\delta}(x, t)+K_{1} t}{\varepsilon}<-\frac{\delta}{4 \varepsilon} \quad \text { for }(x, t) \in\left\{(x, t) ; d_{\delta}(x, t)<\delta / 2\right\} .
$$

Therefore 2.11] yields

$$
\begin{aligned}
\psi_{\varepsilon}(x, t) & =Q\left(\frac{\omega_{\delta}(x, t)+K_{1} t}{\varepsilon}\right)+\varepsilon K_{2} \\
& \leqslant Q\left(-\frac{\delta}{4 \varepsilon}\right)+\varepsilon K_{2} \leqslant-1+C_{1} \exp \left(-\frac{C_{2} \delta}{4 \varepsilon}\right)+\varepsilon\left(K_{2}+C_{3}\right)
\end{aligned}
$$

for $(x, t) \in\{(x, t) ; d(x, t)<-\theta\}$. Combining all the above inequalities, we obtain

$$
v(x, t) \leqslant-1+C_{1} \exp \left(-\frac{C_{2} \delta}{4 \varepsilon}\right)+C \varepsilon \quad \text { for }(x, t) \in\{(x, t) ; d(x, t)<-\theta\},
$$

provided $\varepsilon \in\left(0, \varepsilon_{1}\right)$, where $C_{1}$ and $C_{2}$ are numerical constants, $C=K_{2}+C_{3}$ is a positive constant depending only on $\Lambda_{\beta}$ and $\delta$, and $\varepsilon_{1}$ is a positive constant depending only on $\Lambda_{\beta}, \Lambda_{\gamma}, \Lambda_{f}$ and $\delta$.

\section{Concluding remarks}

We now explain the difference between [ElS2] and our paper and also discuss some remaining problems. We keep our notations $\alpha, \gamma, \beta$ and $f$, which correspond to $A, B, \beta$ and $u$ in [ElS2]. 
(i) (Essential difference) If we assume the driving force $f$ is constant in [EIS2], several propositions of ours have something in common with those of [ElS2], for example our Lemma 3.2 and [EIS2, Lemma 3.3]. The crucial difference is found in the proof of our Lemma 3.3 and [EIS2, Lemma 3.4]. In [EIS2] the authors show that, for $F(p, X)=-\gamma(p)\left\{\operatorname{tr}\left(D^{2} \gamma(p) X\right)+f\right\}$,

$$
F(p, I) \leqslant C\left(\tilde{\Lambda}_{\gamma}\right)(1+|p|) \quad \text { for } p \neq 0,
$$

where $C\left(\tilde{\Lambda}_{\gamma}\right)$ is a constant depending on $\tilde{\Lambda}_{\gamma}:=\|\gamma\|_{C^{2}\left(B_{2}(0) \backslash B_{1 / 2}(0)\right)}$. By using this estimate they prove that $z=z(x, t)$ in the proof of our Lemma 3.3 is a viscosity subsolution of (2.4), and consequently determine $L$. In the case $f$ is a constant, this dependence of $L$ on the second derivatives of $\gamma$ is the crucial reason why the estimate of convergence depends on the derivatives of $\gamma$. In our paper, without using the estimate [6.1, we rather calculate the quantity

$$
F\left(D \gamma^{\circ}(p), D^{2} \gamma^{\circ}(p)\right)=-\frac{n-1}{\gamma^{\circ}(p)}-f \quad \text { for } p \neq 0,
$$

by using convex analysis, and determine the constant $L_{\beta}$ from this formula. Evidently, this calculation is independent of the second derivatives of $\gamma$.

(ii) (Technical difference) There is a difference in the strategies of [EIS2] and ours. In [EIS2] the authors consider the approximation of each problem to clarify the relation of (2.6) and (2.4). In our paper we introduce a modified Allen-Cahn equation (2.9) instead of (2.6) to remove some technical difficulties. However, since we require a detailed estimate rather than the convergence result, we need a more detailed computation.

(iii) (Inhomogeneity) If the driving force $f$ depends on the spatial variables $x$, even if the dependence is $C^{2}$, the method of our paper is not enough to achieve our goal. In fact, in the case $f=f(x)$, the traveling wave $Q$ in $\$ 2.3$ depends on the spatial variable $x$, i.e., $Q=Q(\sigma, x)$. Then we obtain formally

$$
\begin{aligned}
\nabla \psi & =\frac{Q_{\sigma}}{\varepsilon} \nabla \omega+Q_{x}, \\
\nabla^{2} \psi & =\frac{Q_{\sigma \sigma}}{\varepsilon^{2}} \nabla \omega \otimes \nabla \omega+\frac{1}{\varepsilon}\left(Q_{\sigma x} \otimes \nabla \omega+Q_{x \sigma} \otimes \nabla \omega\right)+Q_{x x} .
\end{aligned}
$$

We cannot use the homogeneity of $\alpha, \beta$ and $\gamma$ to estimate $R_{\varepsilon}$ or $\tilde{R}_{\varepsilon}$ because of the form of $\nabla \psi$. Moreover, it is not clear how to estimate the $\varepsilon^{-1}$-term of $\nabla^{2} \psi$. In [EIS2] the authors assume that the highest order derivatives of $\alpha, \beta$ and $\gamma$ are Lipschitz continuous, and calculate that, for example,

$$
D^{2} \alpha(\nabla \psi)=\frac{1}{\varepsilon} D^{2} \alpha(\nabla \omega)+O(1) \quad \text { as } \varepsilon \rightarrow 0 .
$$

The bound of the last term depends on the Lipschitz constant of $D^{2} \alpha$.

(iv) (Time-dependent driving force) It is easy to apply our methods to estimate the internal layer with time-dependent driving force $f(t)$ satisfying, for example, $f \in C^{1}([0, T])$. Essentially, to apply our method for the problem with driving force $f(t)$, we need the following properties:
(a) $Q=Q(\sigma, t), Q^{-1}(\sigma, t) \in C^{2,1}(\mathbb{R} \times[0, T])$,
(b) $\left\|Q_{t}\right\|_{L^{\infty}(\mathbb{R} \times[0, T])}<\infty$,
(c) the convergences as in Proposition 2.1]1], (iii) are uniform with respect to $t \in[0, T]$. 
By the equation (2.10) the traveling wave $Q=Q(\sigma, t)$ with $f \in C^{1}([0, T])$ satisfies the above conditions. In the proof of the propositions, we should be careful with the limiting procedure for sequences of times, in particular, in the proof of Propositions 4.1 and 4.2

Fortunately, when we verify that $\psi_{\varepsilon}$ is a viscosity supersolution of 2.6) and 2.9], this generalization yields only one extra term of the time derivative of $\psi_{\varepsilon}$, i.e.,

$$
\partial_{t} \psi_{\varepsilon}=\frac{Q_{\sigma}}{\varepsilon}\left(\partial_{t} \omega_{\delta}+K_{1}\right)+Q_{t}
$$

The last term is included only in the term of order $\varepsilon^{0}$ of $R_{\varepsilon}$.

(v) (Application for the driving force $f=f(t)$ ) We remark that an application in $§ 2.5$ is still valid for $f=f(t)$ depending on $t$. Suppose that $f_{0}$ is continuous. It is easy to approximate $f_{0}$ by a smooth function $f^{\tau}$ converging to $f_{0}$ locally uniformly. That the convergence ansatz extends to this situation is proved in [GG4] and [GG5]. However, by remark (iv), we need the bound of $\|f\|_{C^{1}([0, T])}$ to verify that our function $\psi_{\varepsilon}$ is a viscosity supersolution of (2.9) by the method developed in this paper. Another method seems to be necessary to prove our uniform convergence for $f=f(t)$ without a bound on $f^{\prime}$.

\section{Acknowledgments}

The work of the first author was partly supported by a Grant-in-Aid for Scientific Research, No. 14204011, the Japan Society of the Promotion of Science and the Grant-in-Aid for formation of COE 'Mathematics of Nonlinear Structures via Singularities' (Hokkaido University). The second author is grateful to Professor Hitoshi Ishii, Professor Tôru Nakajima and Dr. Okihiro Sawada for valuable discussions and advice. The work of the second author was partly supported by the 21 century COE program at Graduate School of Mathematical Sciences, the University of Tokyo.

\section{REFERENCES}

[AC] AllEN, S., \& CAHN, J. A microscopic theory for antiphase boundary motion and its application to antiphase domain coarsing. Acta Metall. 27 (1979), 1084-1095.

[AG] Angenent, S. B., \& Gurtin, M. E. Multiphase thermomechanics with interfacial structure 2. Evolution of an isothermal interface. Arch. Ration. Mech. Anal. 108 (1989), 323-391. Zbl 0723.73017 MR 1013461

[BDL] BARLES, G., \& DA LIO, F. A geometrical approach to front propagation problems in bounded domains with Neumann-type boundary conditions. Interfaces Free Bound. 5 (2003), 239-274. Zbl 1046.35059 MR 1998615

[BSS] BARles, G., SONER, H. M., \& Souganidis, P. E. Front propagation and phase field theory. SIAM J. Control Optim. 31 (1993), 439-469. Zbl 0785.35049 MR 1205984

[BS] Barles, G., \& Souganidis, P. E. A new approach to front propagation problems: Theory and applications. Arch. Ration. Mech. Anal. 141 (1998), 237-296. Zbl 0904.35034 MR 1617291

[BGN] Bellettini, G., Goglione, R., \& Novaga, M. Approximation to driven motion by crystalline curvature in two dimensions. Adv. Math. Sci. Appl. 10 (2000), 467-493. Zbl 0979.53075 MR 1769163

[BN] Bellettini, G., \& Novaga, M. Approximation and comparison for nonsmooth anisotropic motion by mean curvature in $\mathbb{R}^{N}$. Math. Models Methods Appl. Sci. 10 (2000), 1-10. Zbl 1016.53048 MR 1749692 
[BP1] Bellettini, G., \& PAOlini, M. Quasi-optimal error estimates for the mean curvature flow with a forcing term. Differential Integral Equations 8 (1995), 735-752. Zbl $0820.49019 \mid$ MR 1306590

[BP2] Bellettini, G., \& PAOLIni, M. Anisotropic motion by mean curvature in the context of Finsler geometry. Hokkaido Math. J. 25 (1996), 537-566. Zbl 0873.53011 MR 1416006

[BK] BRONSARD, L., \& KOHN, R. Motion by mean curvature as the singular limit of GinzburgLandau dynamics. J. Differential Equations 90 (1991), 211-237. Zbl 0735.35072 MR 1101239

[Ca] CAmilli, F. A stability property for the generalized mean curvature flow equations. $A d v$. Differential Equations 3 (1998), 815-846. Zbl 0957.35072 | MR 1659277

[C] CHEN, X. Generation and propagation of interface in reaction-diffusion equations. J. Differential Equations 96 (1992), 116-141. Zbl 0765.35024 MR 1153311

[CGG1] Chen, Y.-G., GigA, Y., \& Goto, S. Uniqueness and existence of viscosity solutions of generalized mean curvature flow equations. J. Differential Geometry 33 (1991), 749-786. Zbl 0696.35087 MR 1100211

[CGG2] Chen, Y.-G., Giga, Y., \& Goto, S. Analysis toward snow crystal growth. Functional Analysis and Related Topics (Sapporo, 1990), S. Koshi (ed.), Singapore, World Sci. (1991), 43-57. Zbl 0817.76090 MR 1148605

[CIL] Crandall, M. G., Ishit, H., \& LiOns, P.-L. User's guide to viscosity solutions of second order partial differential equations. Bull. Amer. Math. Soc. 27 (1992), 1-67. Zbl 0755.35015 MR 1118699

[DS] DE Mottoni, P., \& Schatzman, M. Geometrical evolution of developed interfaces. Trans. Amer. Math. Soc. 347 (1999), 1533-1589 (announcement: Evolution géométrique d'interfaces, C. R. Acad. Sci. Sér. I. Math. 309 (1989), 453-458). Zbl 0698.35078 MR 1672406

[ElPS] Elliott, C. M., Paolini, M., \& Schätzle, R. Interface estimates for the fully anisotropic Allen-Cahn equation and anisotropic mean-curvature flow. Math. Models Methods Appl. Sci. 6 (1996), 1103-1118. Zbl 0873.35039 MR 1428147

[ElS1] Elliott, C. M., \& SChätZle, R. The limit of the anisotropic double-obstacle AllenCahn equation. Proc. Roy. Soc. Edinburgh Sect. A 126 (1996), 1217-1234. Zbl 0865.35073 MR 1424223

[ElS2] Elliott, C. M., \& SchÄtZle, R. The limit of the fully anisotropic double-obstacle AllenCahn equation in the nonsmooth case. SIAM J. Math. Anal. 28 (1997), 274-303. Zbl 0870.35128 MR 1434036

[ESS] Evans, L. C., Soner, H. M., \& Souganidis, P. E. Phase transitions and generalized motion by mean curvature. Comm. Pure Appl. Math. 45 (1992), 1097-1123. Zbl 0801.35045 MR 1177477

[ES] EvAns, L. C., \& SPRUCK, J. Motion of level sets by mean curvature, I. J. Differential Geometry 33 (1991), 635-681. Zbl $0726.53029 \mid$ MR 1100206

[GG1] GigA, M.-H., \& GigA, Y. Consistency in evolutions by crystalline curvature. Free Boundary Problems, Theory and Applications (Zakopane, 1995), M. Niezgódka and P. Strzelecki (eds.), Longman (1996), 186-202. Zbl 0865.35146 MR 1462981

[GG2] GigA, M.-H., \& GigA, Y. Evolving graphs by singular weighted curvature. Arch. Ration. Mech. Anal. 141 (1998), 117-198. Zbl 0896.35069 MR 1615520

[GG3] GIGA, M.-H., \& GIGA, Y. Stability for evolving graphs by nonlocal weighted curvature. Comm. Partial Differential Equations 24 (1999), 109-184. Zbl 0924.35177| $\mid$ MR 1671993

[GG4] GigA, M.-H., \& GigA, Y. Motion driven by nonlocal curvature in the plane. Asymptotics in Nonlinear Diffusive Systems, Y. Nishiura et al. (eds.), Tohoku Math. Publ. 8 (1998), 75-83. Zbl 0979.35502 MR 1617483

[GG5] GIGA, M.-H., \& GigA, Y. Generalized motion by nonlocal curvature in the plane. Arch. Ration. Mech. Anal. 159 (2001), 295-333. Zbl 1004.35075 MR 1860050 
[G1] GIGA, Y. A level set method for surface evolution equations. Sugaku 47 (1995), 321-340; English transl., Sugaku Expositions 10 (1997), 217-241. Zbl 0898.49015 MR 1600659

[G2] GigA, Y. Surface Evolution Equations-A Level Set Approach. Birkhäuser (2006). Zbl pre05014137

[GGIS] GIGA, Y., Goto, S., IshiI, H., \& S ATO, M.-H. Comparison principle and convexity preserving properties for singular degenerate parabolic equations on unbounded domains. Indiana Univ. Math. J. 40 (1991), 443-470. Zbl 0836.35009 MR 1119185

[GGu] GigA, Y., \& GURTIN, M. A comparison theorem for crystalline evolution in the plane. Quart. Appl. Math. 54 (1996), 727-737. Zbl 0862.35047 MR 1417236

[I] ILMANEN, T. Convergence of the Allen-Cahn equation to the Brakke's motion by mean curvature. J. Differential Geometry 38 (1993), 417-461. Zbl 0784.53035 MR 1237490

[LSU] Ladyzhenskaya, O. A. Solonnikov, V. A., \& URAL'CEVA, N. W. Linear and Quasilinear Equations of Parabolic Type. Amer. Math. Soc., Providence (1967). Zbl 0174.15403 MR 0241822

[MWBCS] McFadden, G. B., Wheeler, A. A., Braun, R. J., Coriell, S. R., \& Sekerka, R. F. Phase field models for anisotropic interfaces. Phys. Rev. E 48 (1993), 2016-2024. MR 1377920

[Sc] SchneIder, R. Convex Bodies; the Brunn-Minkowski Theory, Cambridge Univ. Press (1993). Zbl 0798.52001 MR 1216521

[So] SONER, H. M. Ginzburg-Landau equation and motion by mean curvature, I: convergence, II: development of the initial interface. J. Geom. Anal. 7 (1997), 437-475, 477-491. Zbl 0935.35061 MR 1674800

[T] TAYLOR, J. Constructions and conjectures in crystalline nondifferential geometry. Differential Geometry (Rio de Janeiro), B. Lawson and K. Tanenblat (eds.), Pitman Monogr. Surveys Pure Appl. Math. 52, Pitman, London (1991), 321-336. Zbl 0725.53011 MR 1173051

[TC] TAYLOR, J., \& W. CAHN, J. Diffuse interface with sharp corners and facets: Phase field models with strongly anisotropic surfaces. Phys. D 112 (1998), 381-411. Zbl 0930.35201 MR 1607466

[WM] Wheeler, A. A., \& MCFAdDen, G. B. A $\xi$-vector formulation of anisotropic phasefield models: 3D asymptotics. Europ. J. Appl. Math. 7 (1996), 367-381. Zbl 0909.35160 MR 1413370 